XXVII.

\title{
Das Carcinom und seine Therapie im Lichte der Biologie.
}

\author{
Von
}

Dionys Hellin (Warschau).

\section{I $\mathbf{n} \mathbf{h} \mathbf{a} \mathbf{t}$.}

Einleitung.

Erster Teil.

I. Allgemeine biologische Gesetze: Gegenseitige Abhängigkeit von Lebensdauer, Grösse, Vermehrung, Grad der phyletischen Dignität, Regeneration, Zahl der einzelnen Individuen und Wachstumstempo.

II. Der Einfluss der Nahrung auf die geschlechtliche und die ungeschlechtliche Vermehrung. Jede Vermehrung, sowohl die geschlechtliche wie die ungeschlechtliche, ist immer die Folge von Nahrungsmangel; bei geschlechtlicher Vermehrung wirkt der Nahrungsmangel direkt, bei der ungeschlechtlichen indirekt.

III. Die verschiedene Grösse der Zellen ist abhängig vom Wachstumstempo, folglich auch von Ernährungsbedingungen. Beweise aus der Embryologie.

IV. Die Ursache der grösseren phylogenetischen Dignität der Zellen. Beispiele: Blut-, Epithel- und Nervenzellen.

\section{Zweiter Teil.}

Zellvermehrung durch Reizung. Erklärung der charakteristischen Eigenschaften des Carcinoms: 1. Entstehung infolge von chronischem Reiz, 2. massenhafte Zellvermehrung, 3. Entdifferenzierung, 4. Vorkommen im höheren Alter, 5. Wachstum in die Tiefe, 6. Entwickelung aus dem Epithel.

Ad 1-3. Einfluss der Ernährung auf die Wucherung der Zellen. „Atrophische" Wucherung. Die Hungerversuche von v. Seeland. Syncytioma malignum. Entdifferenzierung bei Actinosphaerium nach Versuchen von Hertwig. Der Basalzellenkrebs. Krebs und Paralytiker. - Der Wucherung geht Untergang von Zellen voraus. Versuche von Ribbert und Lubarsch. - Einfluss von Temperatur, Wasser, Osmose, Alkalien, Licht und Sanerstoff auf das Wachstum überhaupt und speziell beim Carcinom. Ad 3. Das Carcinom ist ein abortiver, neotenischer Prozess. Dädurch wird die massenhafte Zellvermehrung verständlich. Erscheinungen der Entdifferenzierung bei Carcinomen, die in mit Flimmerepithel 
bekleideten Organen entstehen und bei anderen Prozessen: bei Myxosarkoma, im Periost, bei Transplantation, bei Atrophie, in der Narbe, bei Entzündung, in normalen Zellen, in der Schilddrüse; Corpus luteum, Decidua, Eizelle; progressive perniziöse Anämie. Das Carcinom entsteht durch Wirkung des Reizes auf die normale und nicht, wie v. Hansemann meint, nur auf die anaplastische Zelle. Ursache des Unterschiedes zwischen Embryonal- und Carcinomzellen.

Ad 4. Ursache der Prädisposition des höheren Alters für Carcinom: Periodizität inbezug auf das Wachstum, Antagonismus zwischen dem Wachstum einzelner Körperteile. Die Theorie von Thiersch ist nicht ganz richtig: gerade auf der geringen Regenerationskraft des Epithels beruht die Carcinomentwickelung im höheren Alter. Lebercarcinom.

Ad 5. Beispiele von Aenderung der Wachstumsrichtung bei normalen Prozessen. Warum das Carcinom in die Tiefe und nicht gegen diø Oberfläche zu wächst.

Ad 6. Weitere Beweise dafür, dass das Carcinom sich aus dem Epithel und nicht aus dem Bindegewebe entwickelt. Die Rolle des Bindegewebes bei der Carcinomentwickelung.

\section{Dritter Teil.}

Therapie.

Spontane Heilung. Verschiedene Grade von Bösartigkeit inbezug auf die Dauer der Carcinomgeschwulst und inbezug auf die Metastasen. Uebergang von weniger differenziertem Gewebe in ein mehr differenziertes und umgekehrt: Fibrosarkom, Entzündung, Vernarbung, Myxosarkoma, Metastasen, Corpus luteum. Verlangsamung des Wachstumstempos.

Aenderung der Wachstumsrichtung: durch Elektrizität (Fabre-Domergue), durch osmotische Einflüsse, durch Einfluss von Licht, Sauerstoff, durch chemische Mittel. Schädlichkeit der Alkalien beim Carcinom. Versuche von W. A. Freund. Anwendung von Säuren, event. von reduzierenden Mitteln. Einwirkung von Nabrangs- und Wasserentziehung, von Temperaturerniedrigung. Geschlechtsreife. Verlängerung der Lebensdauer bei Pflanzen und Tieren. Mittel, welche lokale Anämie hervorrufen. Eingriffe an verschiedenen Körperteilen. Vernarbung des Carcinoms. Therapeutische Versuche mit Nervengewebe.

\section{Einleitung.}

"Man hat nur Sinn für Detailforschungen und ihnen gibt man sich mit dem unermüdlichsten Fleisse hin und bedenkt dabei nicht, dass es sich in der Wissenschaft nicht allein darum handelt, die Bausteine an das Tageslicht zu fördern, sondern dass dieselben auch zusammengefügt werden." "Man fürchtet sich vor Systemen, weil man dabei immer an die früheren philosophisch-spekulativen Systeme denkt, und übersieht doch, dass auch jede empirische Wissenschaft ihr System haben muss" (A. Spiess in Virchows Archit. Bd. 8. S. 341).

„Bald von dieser, bald von jener Seite wird gerufen: nun sei es doch genug der Tatsachen; man möge auch wieder ordnen, auf dass man wisse, wo Alles hin- 
gehöre" (Virchow in seinem Archiv, Bd. 8. S. 5). "Spekulation ist sowohl bei der Verwertung der pathologisch-anatomischen als bei derjenigen der pathologisohphysiologischen Tatsachen notwendig: in beiden Fällen müssen die Erscheinungen kombiniert abgeleitet werden" (Virchow in seinom Archiv, Bd.6. S. 19). - Man soll: „animo contemplare, quod oculo non vides." "Denkende Erfahrung" bestimmt, nach v. Baers Ausdruck, den Weg für den Naturforscher.

"Wie die Betrachtung Ursache eines Experimentes ist, so wird sie auch zu dessen Folge" (Kronthal, Von der Nervenzelle etc. 1902. S. 44).

„Nachdem die Philosophie eine Weile vorherrschend gewesen, verfällt sie für eine lange Zeit der Vernachlässigung, um sich dann allmählich wieder zu erheben. Jede Wissenschaft hat ihre Epochen der deduktiven Behandlungsweise und Epochen, wo die Aufmerksamkeit hauptsächlich auf das Sammeln und Zusammenstellen von Tatsachen gerichtet ist" (Spencer, Philosophie, S. 273).

„Die Rolle der Wissenschaft besteht darin, dass sie die Fragen anders stellt, indem sie dieselben einige Schritte vorwärts schiebt, allmählich vereinfacht und, von einer sehr allgemeinen Ursache ausgehend, zu lösen hilft" (Sabatier, Leben und Tod).

Da ich bei meinen Studien über Carcinom auf Veröffentlichung in einer einzigen Abhandlung aus vielen Gründen von vornherein verzichten zu müssen mich gezwungen sah, erscheinen meine Publikationen darüber nur etappenweise. Ich verweise hier auf meine erste Abhandlung ${ }^{1}$ ) und bemerke, dass im Laufe der seit dieser Publikation verflossenen Zeit ich zur Aenderung meiner dort geäusserten Deduktionen und Analysen nicht nur keine Veranlassung sehe, sondern im Gegenteil haben die Arbeiten während dieser Zeit eine weitere Stütze für meine Ansichten geliefert. Was damals nur bescheiden ausgesprochen wurde, kann jetzt mit einer gewissen Bestimmtheit hervorgehoben werden. Es galt ja nicht nur bereits statuierte biologische Gesetze auf das Studium des Carcinoms zu übertragen, sondern auch manche neue Gesetze mussten eruiert werden. In dieser Hinsicht war die Arbeit nicht nur speziell für Pathologen, sondern auch im allgemeinen für Biologen nicht ohne Wert. Eben diese neuen Gesetze fanden vielfache Bestätigung in den seither publizierten Arbeiten ${ }^{2}$ ).

In unserer Zeit, wo nur das, was unmittelbar praktische Anwendung erlangt, besonderen Anklang findet, haben solche Untersuchungen, die sich an allgemeine biologische Fragen anlehnen, wenig Aussicht auf weite Verbreitung, weil die Frage über den Krebs hauptsächlich den Praktiker interessiert; und es würde viel Mühe kosten, unter den jetzt am meisten bekannten Praktikern, wenn wir z. B. die klinischen Fächer der medizinischen Fakultäten ins Auge fassen, jemanden $z \mathfrak{u}$ finden, der sich mit allgemein biologischen Fragen besehäftigen würde. Doch diese Ueberlegungen können niemand abhalten, seines Weges zu gehen. Mit der Zeit, wenn auch

1) Das Carcinom, eine biologische Skizze. Leipzig 1898. F. C. W. Vogel. 2) ef. Anm. 3, S. 598. 
langsam, müssen und werden sich neue Richtungen Bahn brechen. Dass sich auf diesem Wege doch etwas erreichen lässt, habe ich durch meine Abhandlung: "Die Ursache der Multiparität der uniparen Tiere überhaupt und der Zwillingsschwangerschaft beim Menschen insbesondere" 1 ) bereits bewiesen, indem ich zeigte, dass seit jeher bekannte Tatsachen, zu biologischen Gesetzen verallgemeinert, genügten, um die bis dahin unbekannte Ursache der Entstehung von Zwillingsschwangerschaft zu ergründen. Erlaubt es mir die Zeit, so werde ich später beide Prozesse: Carcinom und Zwillingsschwangerschaft, von für beide gemeinsamen biologischen Gesetzen ausgehend, beleuchten. Derjenige, der die genannten Arbeiten gelesen hatte, wird aus ihnen bereits ersehen haben, dass der leitende Gedanke, das Prinzip der Erklärung im wesentlichen dasselbe ist. Das Bestreben unserer Wissenschaft, ihr Endziel, ist eben die Verallgemeinerung der Gesetze, und wenn es mir nur für zwei derartige sogenannte pathologische Erscheinungen geJungen ist, eine Verallgemeinerung zu erreichen, so bin icb damit nicht unzufrieden.

Die Praktiker dürfen nicht so herablassend die biologischen Forschungen behandeln. Ihnen, denen es hauptsächlich um die Therapie zu tun ist, muss auch die biologische Forschung willkommen sein. Von der Erkenntnis dessen, wie, unter welchen Bedingungen etwas entsteht, ist der Weg nicht so weit zu der Erkenntnis: wie wirkt man der Entstehung und der Verbreitung dieses Phänomens entgegen, was auch tatsächlich in meiner früheren Publikation und auch in der jetzt vorliegenden besprochen wird. Von einer so komplizierten Frage, wie es das Carcinom ist, darf man aber nicht erwarten, dass sie so bald gelöst werden wird. Allein die Hoffnung erscheint uns als immer mehr und mehr erfüllbar, natürlich wenn man nur nicht "le pourquoi di pourquoi" wissen will. Man darf von der Wissenschaft nicht mehr verlangen, als dies unter den obwaltenden Verhältnissen möglich ist, denn auch sie, „obwohl die schönste unter den schönen, kann nicht mehr geben, als sie selbst hat."

Der etwaige Vorwurf, dass meine Untersuchungen rein theoretisch seien, wird dadurch zurückgewiesen, dass alles, was hier ausgeführt wird, als Schluss aus Tatsachen nur der Form nach theoretisch ist; es ist aber keine blosse Spekulation, sondern alles stützt sich auf anatomische, speziell auf pathologisch-anatomische und auch pathologisch-physiologische Tatsachen. Nun wird man aber sagen, dass, wenn nur bekannte Tatsachen angeführt werden, die Arbeit nichts Neues bieten kann. Allein Gesetze, die neu aufgestellt werden, stützen sich jedesmal auf altbekannte Tatsachen und eben die neuen Gesetze sind es, die die Wissenschaft ausmachen, nicht die puren Tatsachen.

1) München 1895. Verlag von Seitz u. Schauer. 
Der jetzt vorliegenden Abhandlung wird voraussichtlich im Laufe der nächsten secbs Jahre eine weitere folgen, in der ich u. a. auf Grund von Studien über das Chorioepithelioma malignum einen Schritt weiter in der Frage über das Carcinom zu machen die Hoffinung hege.

\section{Erster Teil.}

\section{Allgemeine biologische Gesetze ${ }^{1}$ ).}

A.

a) Lebensdauer (und die Dauer der einzelnen Lebensperioden).

b) Phylogenetische Dignität.

c) Grösse.

a) Vermehrung.

B.

b) Regeneration.

c) Zahl der einzeluen Individuen ${ }^{2}$ ).

d) Wachstumstempo.

Die unter A verzeichneten Faktoren (ebenso die unter B verzeichneten untereinander) sind einander direkt, jeder von den unter A verzeichneten ist den unter B aufgezählten umgekehrt proportional oder mit anderen Worten: Das Produkt aus der Lebensdauer oder der Grösse eines Organismus und seiner Progenitur ist eine konstante Grösse. Man kann also, wenn die eine von diesen Grössen bekannt ist, die zweite durch blosse Division berechnen ${ }^{3}$ ).

1) Diese Gesetze gelten sowohl für einzelne Individuen des Tierreiches, für einzelne Stämme, Klassen, Ordnungen, Familien, Gattungen und Arten desselben, wie auch für einzelne Zellen eines und desselben Organimus. Auch für die Pflanzenwelt gilt im allgemeinen dasselbe.

2) Klassen und Ordnungen im Tier- und Pflanzenreich, deren Organisation am wenigsten kompliziert ist, zeigen stets die weiteste Verbreitung (Hooker). Die Zahl der grösseren und höher entwickelten Zellen jm Organismus ist geringer, als die der kleineren. Man vergleiche nur z. B. Epidermis- und Nervenzellen. Während die ersteren eng beieinander liegen, rücken die letzteren, den Verband gänzlich aufgebend, auseinander, denn auch in Spinalganglien, wo sie am dichtesten gedrängt stehen, grenzt bekanntlich niemals eine Zelle an die andere, und im Zentralnervensystem ist sogar meist ein beträchtlicher Zwischenraum zwischen den einzelnen Zellen zu finden. Die Länge einer Nervenfaser, über die sich der trophische Einfluss ihrer Zelle erstreckt, beträgt oft über einen Metèr. - Diesem Gesetz entsprechend sind die Zellen in der vorderen Hälfte der Keimscheibe grösser, aber in geringerer Zahl, hinten sind sie kleiner und zahlreicher.

3) Dieses Gesetz erinnert an das D ulong-Petit'sche in der Chemie, nach welchem das Prodult aus Atomgewicht und spezifischer Wärme eine konstante 


\section{Einfluss der Ernährung auf die Vermehrung.}

Jede Vermehrung, sowohl die ungeschlechtliche wie die geschlechtliche, stellt nur verschiedene Formen einer und derselben Erscheinung vor, nämlich des Wachstums: die Vermehrung ist nur „ein Wachstum über die Schranke des Individnums hinaus" (v. Baer $\left.{ }^{1}\right)$ ). Die Vermehrung kommt sowohl infolge ron Nahrungsüberfluss als Nabrungsmangel zustande, und zwar entsteht, wie bekannt, z. B. bei denjenigen Tieren, die sich sowohl auf geschlechtlichem wie ungeschlechtlichem Wege vermehren, durch Nahrungsüberfluss eine Steigerung der Produktivität auf ungeschlechtlichem Wege, bei Nahrungsmangel auf geschlechtlichem Wege ${ }^{2}$ ). Jeder Vermehrung - sowohl der geschlechtlichen wie der ungeschlechtlichen - liegt immer die Erzielung einer grösseren, günstigeren Ernährungsfäche zu Grunde, denn durch Teilung wird die Oberfläche vergrössert.

Dass dem so ist, weiss man schon lange, aber wie es möglich ist, dass $z$ rrei entgegengesetzte Faktoren - Nahrungszufuhr und Nahrungsmangel - eine und dieselbe Wirkung, nämlich Vermehrung, hervorrufen können, diese Frage hat nicht nur niemand bis jetzt gelöst, sondern man hat bis jetzt überhaupt auf diese Kontroverse nicht aufmerksam gemacht. Die Erklärung liegt aber sehr nahe:

a) Beim Nahrungsmangel tritt deshalb geschlechtliche Vermehrung, d. h. die Abtrennung vom Organismus ein, weil bei gemeinsamer Existenz die Nachkommenschaft ebenfalls an Nahrungsmangel leiden und so zu Grunde gehen würde. Der Zweck (die Ursache) der geschlechtlichen Vermehrung ist ja eben auf dem Wege der Trennung vom mütterlichen Organismus durch auf diese Weise erzielte Vergrösserung der Oberfläche günstigere Bedingungen für die Ernährung zu gewinnen; die Befruchtung ist somit nichts anderes als ebenfalls eine Art Ernährung, eine besondere Form von Nabrungsaufnahme, eine sehr rasch sich assimilirende Ernährung, indem die Ernährungselemente unmittelbar in das zu ernährende Wesen gelangen, um sich rasch zu assimiliren, ohne umgeändert werden zu müssen ${ }^{3}$ ).

Grösse, ungefähr $=6,5$ ist. All diese Konstanten lassen sich durch eine gleichseitige Hyperbel laut $\mathrm{xy}=$ konst graphisch darstellen. - Ausnabmen sowohl in der Tier- und Pflanzenwelt wie in der Chemio erklären sich durch den Einfluss sekundärer Faktoren. Ich kann hier leider nicht näher darauf eingehen.

1) Es hat, wie wir sehen, v. Baer schon vor $\mathrm{Haeckel}$ diesen Gedanken ausgesprochen.

2) Cf. Spencer, Biologie.

3) Delboeuf (cf. Sabatier, La vie et la mort). - Es besteht also ein enger Zusammenhang zwischen Hunger und Liebe. 
b) Ist Nahrungsüberfluss vorhanden, so tritt ungeschlechtliche Vermehrung auf. Da jede Vermehrung aber, somit auch die ungeschlechtliche, den $Z$ weck hat, eine grössere Oberfläche behufs besserer Ernährung zu erlangen, also jedesmal ein Zeichen von Nahrungsmangel ist, so stossen wir hier auf einen Widerspruch (der aber nur scheinbar ist): wie kann ungenügende Ernährung bei genügendem Nahrungsvorrat vorbauden sein, mit anderen Worten: wie kann Nahrungsmangel bei Nahrungsüberfluss existieren? Dieser scheinbare Gegensatz erklärt sich sehr einfach. Die Zelle wächst dank der Nahrungszufuhr. Es wächst dabei ihre Oberfläche und ihre Masse. Die Masse wächst aber schneller als die Oberfläche, indem sie im Verhältnis des Kubus des Durchmessers, die Oberfäche aber im Verhältnis des Quadrates des Durchmessers zunimmt ${ }^{1}$ ). Es muss also ein Moment kommen, wo die Oberfläche zu klein wird im Vergleich mit der Masse, d. h. wo die Zelle keine genügende, ihrer Masse entsprechende Nahrung, auch beim Vorhandensein derselben, aufnehmen kann und wo also - trotz Vorhandensein von Nahrung - Nahrungsmangel eintreten und die Zelle sich teilen muss. Wenn z. B. eine Zelle noch einmal so gross geworden ist, so wird dabei die resorbierende Oberfläche nur vervierfacht, während ihr Inhalt verachtfacht wurde; die Zelle wird also in Bezug auf Aufnahmefähigkeit nur halb so gut stehen, wie vorher. Entsteht somit beim Nahrungsüberfluss im Organismus infolge von mit der Zeit beim Wachstum der Zelle eingetretenem Missverhältnis zwischen Oberfläche und Masse lokal in einer Zelle Nahrungsmangel, so teilt sich die Zelle, aber die neu entstandenen Zellen werden nach der Teilung den Mutterorganismus nicht verlassen, sondern im Kontakt mit ihm bleiben; denn dabei bleibt im Organismus als Ganzem die Nahrung in genügender Menge vorhanden, und sie kann jetzt bei der durch die Teilung erfolgten Vergrösserung der Zellenoberfläche auch in genügender Weise aufgenommen werden. Es führt also Nahrungsüberfluss zum lokalen Nahrungsmangel in den Zellen. Die Vermehrung, sowohI die geschlechtliche wie die ungeschlechtliche, beruht mithin jedesmal unmittelbar auf einer mangelhaften Ernährung. Dieses von mir zum erstenmal statuierte Gesetz ${ }^{2}$ ) wird durch neuere Untersuchungen vollkommen bestätigt ${ }^{3}$ ).

1) Das analoge Gesetz von Malth u s lantet: Die Individuen vermehren sich in geometrischer, während die ihnen als Ernährung dienenden Substanzen sich in arithmetischer Reihe vermehren.

2) Das Carcinom. Leipzig 1898.

3) Pawlin off, Sauerstoffmangel als Ursache des Wachstums usw. Berlin 1902. - Mühlmann, Die Ursache des Alters. Wiesbaden 1900. - Fried. mann, F., Die Altersveränderungen. 1902. - Jickeli, Die Unvollkommenheit des Stoffwechsels als Veranldssung für Vermehrung, Wachstum, Differenzierung usw. 


\section{Ursache der verschiedenen Grösse der Zellen.}

Warum sind nicht alle Zellen gleich gross, wenn sie doch alle von einer Zelle abstammen? Wie kommt der Unterschied in der Grösse der Zellen zustande?

Die Ernährungsbedingungen sind für verschiedene Zellen eines und desselben Organismus verschieden. Es sind drei Möglichkeiten vorhanden: Nahrungsmangel, Nahrungsüberfluss, Nahrungsgleichgewicht (d. h. die Zufuhr von so viel Nahrung, dass diese nur die Ausgabe deckt, laut Verworn's Formel $\frac{A}{B}=1$ ). a) Ist Nahrungsmangel in einer Zelle vorhanden, so muss sie sich sofort teilen, damit sie nicht zu Grunde gehe. Von zwei Stellen, von denen an einer Nahrungsüberfluss, an der anderen Nahrungsmangel herrscht, wird natürlich diejenige sich schneller teilen, wo Nahrungsmangel ist, weil ihr unmittelbar der Tod droht. b) an der nahrungsreichen Stelle werdeh die Zellen verhältnismässig rasch eine Volumsvergrösserung eintreten lassen; das daraus resultierende Missverhältniss zwischen Oberfläche und Masse führt zur Teilung der Zelle, d. h. zur Erlangung einer im Verhältnis zur Masse grösseren Oberfäche. Die Teilung der Zelle erfolgt also rasch dort, wo Nahrungsmangel oder bedeutender Nahrungsüberfluss vorhanden ist. c) Langsames Wachstum bzw. ein Stehenbleiben auf derselben Grössenstufe kommt da vor, wo die Nahrungszufuhr beinahe nur ausreicht, um-die Ausgaben zu decken. Während dort, wo Nahrungsüberfluss sehr bedeutend ist, die Zelle schnell wächst und sich bald teilen muss, also ohne eine bedeutende Grösse erreicht zu haben, kommt die Inkongruenz zwischen Wachstum der Masse und der Oberfläche in dem Fall, wo kein Nahrungsüberfluss vorhanden ist, erst relativ spät zum Vorschein und die Zelle ist auf diese Weise imstande, eine grössere Oberfläche $\mathrm{zu}$ erlangen. Die Oberfläche kann um so grösser werden, je langsamer die Masse wächst. Das langsame Wachstum trifft aber bei der Bedingung des ungefähren Nahrungsgleichgewichtes ein, d. h. dann, wenn sich die Ernährungsbedingungen dem Wert $\frac{\mathrm{A}}{\mathrm{B}}=1$ nähern.

Durch diese Differenz im Wachstumstempo der Zellen, die ihrerseits durch die Differenz in Ernährungsverhältnissen zustande kommt, tritt eben die Differenz in der Grösse der Zellen ein. Die Ewbryonalentwickelung bietet uns vielfache Beweise in dieser Hinsicht. So sind die medialen Lagen im Embryo die nahrungsärmeren, die lateralen die nahrungsreicheren,

Berlin 1902. - Hertwig, Ueber physiologische Degeneration bei Actinosphaerium Eichborni nebst Bemerkungen zur Aetiologie der Geschwülste. Jena 1904. Brunton, Lancet 1905 . Oct. 14 . p. 1087. 
indem von der Peripherie des Eies mehr Nahrung als von der Mitte aufgenommen wird; aber auch das Wachstum ist in der Mitte ein rascheres als in der Peripherie (Baer, S. $173 \mathrm{ff}$.). Als im späteren Verlauf der Entwickelung die linke Seite des Embryo im Verhältnis zur rechten die aufnehmende wird, bildet sich in der Tat auch die rechte Seite etwas rascher aus. Die Furchung an der sog. vorderen Hälfte der Keimscheibe verläuft langsamer als hinten - vorn sind aber auch die Zellen grösser und von geringerer Zahl, hinten sind sie kleiner und zahlreicher. Im Ei ist die Segmentation da grösser, wo weniger Dotter vorhanden ist; wo er reichlicher vorhanden ist, dort ist auch die Entwickelung geringer. Die Gesetzmässigkeit dieser Erscheinung findet hier ihren Ausdruck in der Existenz eines sogar proportionalen Verhältnisses. Dementsprechend tritt das an Dotter reiche Parablast erst später auf ${ }^{1}$ ). Die dotterärmeren, d. h. protoplasmareicheren (animalen) Zellen teilen sich also rascher als die dotterreicheren (vegetativen) (die Schnelligkeit der Furchung ist proportional der Konzentration des im Teilungsstück befindlichen Protoplasma), sie sind aber auch kleiner als die letzteren. Die Grösse der Zellen hängt also, dem allgemeinen Gesetz entsprechend (Kap. I) vom Tempo des Wachstums, dieses aber von der lokal vorhandenen Nahrungsmenge ab.

\section{Entstehung der höheren phylogenetischen Dignität der Zellen.}

Das langsame Wachstum ist eine Conditio sine qua non für die Erlangung einer höheren Entwickelungsstufe. Nur bei langsamem Wachstum sind Zeit und Möglichkeit zur Ausbildung zweckentsprechender konservierender Eigenschaften gegeben. Da aber langsames Wachstum nur beim Fehlen von Nahrungsüberfluss möglich ist, so folgt daraus, dass die höhere Entwickelung, die Differenzierung nur bei nicht zu reichlicher Nahrungsaufnahme möglich ist. Dementsprechend verringert sich das Wachstumstempo, der Wachstumsrhythmus mit steigender Grösse des Organismus, was sowohl auf Zellen wie auf ganze tierische (und auch pflanzliche) Organismen sich bezieht. Die Vermehrung der Zellen in jedem Organismus weist vom Beginn der Entwickelung an eine immer weniger steil aufsteigende Kurve auf 2). Die ersten vier Wochen der Embryonalentwickelung leisten Grösseres als später ebenso viele Monate (Gegenbaur). Der Embryo wächst sehr rasch, das Kind weniger rasch, der Jüngling verhältnismässig noch langsamer, bis das Wachstum aufzuhören anfängt und im grossen ganzen zum Stillstand kommt. Aus der Bildungsart der Muskelzellen

1) Vergl. Hertwig, Archiv für mikroskop. Anatomie. 1898. - Patten, Zoolog. Anzeiger. 1894. - Journal of Morphology. 1891. Vol. XII.

2) Man rergleicht gewöhnlich dieses Phänomen mit der Kugel, die nach dem Schuss allmählich an Schnelligkeit abnimmt. 
bei niederen Tieren, aus der Lage der Muskelzellen des menschlichen Körpers am Mesoderm schliesst Mühlmann auf das Vorhandensein von Nabrungsmangel dort: die Nuskelfaser entsteht an dem von Nahrungszutritt ab- d. h. an der der Stützlamelle zagewandten Seite ${ }^{1}$ ). Ebenso wird die Bildung des Bindegewebes von Ausscheidung einer Interzellularsubstanz begleitet, eines Produktes der Zellen; diese aber bildet sich zwischen zwei bei einander liegenden Zellschichten überall, wo die Ernährung gestört ist. Thre Entstehung wird durch rückschreitende Metamorphose des Protoplasmas der Zellen bewerkstelligt. Bei den Wirbeltieren findet die reichlichste Bildung der Interzellularsubstanz am medialen Rand der Urwirbel statt, da, wo diese an die Chorda grenzen (welche selbst frühzeitig der Bindegervebsmetamorphose verfällt). Da die Zellen dabei nicht absterben, so ist das - sagt Mühlmann - ein Beweis, dass die Nahrungsaufnahme zwar geringer wurde, aber noch genügend für die Existenz der Zellen ist ${ }^{2}$ ). Die Blutzellen reihen sicb den am wenigsten differenzierten Zellen unseres Organismus an, wofür auch ihre an niedere, einzellige Organismen erinnernde Eigensehaften (z. B. Beweglichkeit) sprechen, sie entstehen in der frühesten Embryonalperiode und gehören zugleich zu den kleinsten Zellen des Organismus; dementsprechend gehen sie rasch zu Grunde und besitzen eine grosse Regenerationskraft. Epithelzellen vermehren sich ebenfalls rasch, treten frühzeitig auf und leben verhältnismässig sehr kurz. Demgegenüber haben die Ganglienzellen die grösste Lebensdauer, sie vermehren sich aber auch kaum, sie wachsen im fertigen Organismus nicht mebr ${ }^{2}$, sie regenerieren nicht, und hat der Organismus einmal eine Ganglienzelle eingebüsst, so ist und bleibt die Zahl seiner Ganglienzellen ein für alle Mal geringer als zuvor. Diese grössten Zellen unseres Organismus treten auch am spätesten von allen anderen während der Embryogenese auf; sie gehören aber zu den höchst entwickelten Zellen unseres Organismus. Wir sehen auch, dass die Grösse des Stoffumsatzes in der Nervenfaser, auch während des Erregungszustandes, sehr gering ist. Es ist bisher nicht unzweifelhaft sicher gelungen, in einer erregten Nervenfaser eine chemische Veränderung oder eine Wärmeentwickelung nachzuweisen, die auf einen erheblichen Stoffumsatz hindeutete. Wie gering der Stoffumsatz erregter Nervenfasern sein muss, geht noch besonders daraus hervor, dass man eine Nervenfaser stundenlang mit künstlichen Reizen irritieren kann, olıne eine merkliche Ermüdung wahrzunehmen ${ }^{3}$ ). Die hohe Dignität der Ganglien-

1) Mühlmann 1. c. (S. $82,151,66)$. - Schneider, Archiv f. mikroskopische Anatomie. 1890. Bd. 35 .

2) F. Schenck, Physiologische Charakteristik der Zelle. Würzburg 1899.

3) Klebs, Allgemeine Pathologie. S. 564. 
zellen geht auch daraus herror, dass eine Ganglienzelle auch keine Zelle mebr im eigentlichen Sinne des Wortes, sondern vielmehr ein Primitivorgan ist, ähnlich dem Nierenglomerulus oder Eifollikel; in Form eines Lymphraumes besitzen die Ganglienzellen besondere Ernährungseinrichtungen $\left.{ }^{1}\right)^{2}$ ).

\section{Zweiter Teil.}

Seit Bichat und Laënnec, besonders aber seit Virchow wissen wir, dass die sogenannten pathologischen Vorgänge denselben Gesetzen gehorchen, wie die physiologischen. Die Natur kennt keine Pathologie, und wir wollen nun zeigen, dass in der Tat alles, was uns im ersten Teil die Biologie gelehrt hat, uns zugleich die Erklärung für die Erscheinungen beim Krebs in die Hand gibt.

Auf den ersten Blick scheint es, dass unsere Kenntnisse über den Krebs sehr gering sind. Ich meinerseits glaube, dass wir nur in Einzelheiten weiterer Auskünfte bedürfen, im grossen und ganzen hat das, was wir bereits über den Krebs wissen, die Grenze des Erwünschten erreicht. Leberblicken wir, worauf die Eigentümlichkeiten des Carcinoms beruhen, so werden wir uns überzeugen, dass sich alles durch allgemeine biologische Gesetze, durch die Gesetze, die auch für andere Prozesse gelten, erklären lässt. Folgende Fragen kommen hier in betracht:

1. Die Entstehung infolge von chronischem Reiz.

2. Die massenbafte Vermehrung der Zellen.

3. Gibt es im Organismus dem Carcinom analoge Prozesse, sowohl normal wie pathologisch?

4. Das Vorkommen im höheren Alter.

5. Warum nicht alle ältere Individuen an Krebs erkranken?

6. Das Wachstum in die Tiefe.

1) Merkel, Verhandl, des X. internat. Kongresses zu Berlin. 1890. IГ. Bd. Abt. 1. S. 130.

2) Aus der Tatsache, dass die Zahl der Ganglienzellen in einem gleich grossen Hirnrindenstück bei höheren Säugern kleiner als bei niederen ist, schliesst Bethe (Allgemeine Anatomie und Physiologie, Jena 1903, S. 76), dass den Ganglienzellen nicht die hohe Bedeutung für die nervösen Prozesse zukommen kann, die ihnen zuerkannt wird. Dieser Schluss ist ganz falsch. Bethe bält irrtümlich eine Verminderung der Zellenzahl für ein Zeichen einer niedrigeren Entwickelungsstufe. Im Gegenteil, gerade eine Verminderung der Zahl mit gleichzeitiger Vergrösserung des Volumens ist, laut unseren Gesetzen (s. S. 596, Anmerk. 2), das Zeichen einer höheren Differenzierung. Und das absolute Mass der Ganglienzelle ist bei den höher entwickelten Tieren in der T'at grösser als bei den auf einer niedrigen Entwickelungsstufe stehenden. 
7. Aus welcher Gewebsschicht entwickelt sich das Carcinom?

8. Wie lange muss der Reiz einwirken, damit ein Carcinom entstehe?

9. In welcher Richtung ist die Therapie zu suchen?

Ad 1 und 2. Wir haben bereits im ersten Kapitel gesehen, dass bei direktem Nahrungsmangel Zellenvermehrung eintritt. Aber nicht nur Nahrungsmangel, sondern auch andere Schädigungsarten der Zellen sind im Stande, eine Vermehrung derselben hervorzurufen. So legen Schmetterlinge nicht selten gleich nach dem Auskriechen aus der Puppenhülle Eier, vorzüglich aber, wenn man sie aufspiesst, oder wenn sie in ganz engen Behältnissen gehalten oder sonst belästigt werden ${ }^{1}$ ). Durch Behandlung mit den verschiedensten Giften (z. B. $\mathrm{HgCl}_{2}, \mathrm{H}_{2} \mathrm{SO}_{4}$, Alkaloide) kann man sowohl bei Tieren wie bei Pflanzen (Seidenspinner, Frosch, Feuerbohne, Hefezelle usw.) ein Wachstum der Keime hervorrufen ${ }^{2}$ ). Die Gebrüder Hertwig (und Boveri) haben gezeigt, dass geschädigte Eier ein grösseres Verlangen nach dem Sperma zeigen und deshalb sich auch bastardieren lassen, wenn sie das sonst nicht tun. So finden wir auch eine Vermehrung der Zellkerne, wenn der spezifisch fungierende Teil in den Muskeln atrophiert ["atrophische Kernwucherung" Cohnheim³)]. Bei dem progressiven Muskelschwund geht gewöhnlich eine Hypertrophie der Fasern und eine Vermehrung der Muskelkerne der später folgenden Atrophie voraus4). So auch beim Typhus. Das Gleiche ereignet sich nach Flemming in den Zellen des atrophischen Fettgewebes ${ }^{5}$ ): die Kerne der Fettzellen vermehren sich bei starker, andauernder Nahrungsentziehung. Der Atrophie des Corpus luteum geht eine Zellvermehrung in demselben voraus.

Fischer und Schiefferdecker haben im Jahre 1870 nachgewiesen, dasș bei Schädigungen der Nerven durch Schussverletzungen die epidermoidalen Gebilde, wie Nägel und Haare, stärker wuchsen, um aber nachher zu atrophieren. So endet diese Hyperplasie mit Atrophie, die Zellen müssen, wie wir bereits bewiesen haben (Kapitel II), unter solchen Umständen zu Grunde geben, nämlich infolge von Vermebrung beim Nahrungsmangel.

1) Baer, S. 151 .

2) Dewitz, Biol. Zentralbl. VII. S. 94. - Mosso, Naturwiss. Rundschau. 1894. S. 581. - Bjernacki, Pflüger's Arch. Bd. 49. - O. und R. Hertwig, Experimentelle Untersuchungen über die Bedingungen der Bastardbefruchtung. Jena 1885.

3) Allgemeine Patbologie. Bd. I. S. 503.

4) F. Schultze, Ueber den mit Hypertrophie verbundenen progressiven Muskelschwund und ähnliche Krankheiten. 1886.

5) Arch. f. mikrosk. Anatom. VII. S. 32, 328; ib. Bd. XII. - Virchow's Arch. Bd. 52. S. 568 . 
Anders würde sich der Prozess gestalten, wenn die Zellen, nachdem sie sich infolge von Nahrungsmangel vermehrt hatten, jetzt unter günstige Ernährungsbedingungen geraten würden. v. Seeland ${ }^{1}$ ) hat experimentell an Tauben und Hühnern nachgewiesen, dass diejenigen von den untersuchten Tieren, die von Zeit $z u$ Zeit gehungert haben und nach jedesmaliger Fastenperiode ebenso reichlich wie die Kontrolltiere gefüttert wurden, mehr wogen, als die normal gefütterten Tiere. Die schädigende Wirkung des Fastens rief hier, nach Jickeli, eine Zellvermehrung hervor, und dank dem Vorhandensein von einer grösseren Anzahl von Zellen konnte auch eher das Gewieht des Versuchstieres bei reichlicher Ernährung zunehmen. Es liesse sich vielleicht dieses Verbalten auch zu therapeutischen $Z$ wecken beim Menschen rerwenden. Vielleicht beruhen die Erfolge bei der Schrottschen Kur zum Teil darauf. Somit findet auch das von den meisten Religionen vorgeschriebene Fasten eine gewisse medizinische Begründung.

Wir haben bereits im vorigen Kapitel gesehen, dass auch Nahrungsüberfluss eine Zellvermehrung hervorrufen muss, indem er auf indirektem Wege zum Nahrungsmangel führt. Während aber bei direktem Nahrungsmangel die sich vermehrenden Zellen bald zu Grunde gehen müssen, führt der Nahrungsüberfluss (d. h. indirekt als Nahrungsmangel) zu fortdauernder Zellwucherung, indem stets neues Material zugeführt wird. So erklärt auch Kworostansky2) die Ursache der Zellwucherung bei Syncytioma malignum. Nach ihm wird dabei der Hämoglobingehalt des Blutes vermindert, hierdurch findet das Chorionepithel an der Oberfläche der Dezidua nicht genügend Nährmaterial und gerät deshalb in starke Wucherung. Und da das Epithel von Natur schon auf parasitäres Leben angewiesen ist, so kriecht es tiefer in die Muskulatur des Lterus hinein und zerstört seine Wände, wie eine Pflanze, die auf Granit wächst und ihre Wurzeln tief in den Boden hinein sendet. So wird der Zufluss von grösserem Nahrungsvorrat erzielt ${ }^{3}$ ). Nach Gottschalk ${ }^{4}$ ) entsteht das Chorioepithelioma malignum, sobald die zur Blasenmole degenerierten Zotten reichlicher von mütterlichem Blut umspült werden (was im Bereich des Chorion laeve im Allgemeinen erst mit der Zerstörung der Kapsularis ermöglicht wird). Das Chorioepithelioma kommt aber nicht nur beim Weibe, sondern auch im Hoden vor, wie wir bereits aus mehreren in der Literatur ange-

1) Biol. Centralbl. VII. 1887. Aehnliche Versuche mit gleichem Resultat sind von Manassein (1869), Kagan (1886), Sadowienj, Albicki, Paschutin (1895) veröffentlicht worden (russ.).

2) Archiv f. Gynäkologie. 1900. LXII. 1. S. 69.

3) Vergl. auch die Versuche ron v. Seeland.

4) Zeitschr. f. Geburtsh. u. Gynäkol. LIII. 3. S. 516. 1904. 
gebenen Fällen wissen, und darin dokumentirt sich die Verwandtschaft des Chorioepithelioma mit anderen malignen Neubildungen. Die Abhängigkeit der Krebswucherung von lokalem Nahrungsüberfluss ergibt sich auch aus dem foudroyanten Fortschreiten des Uteruskrebses in der Schwangerschaft. Hertwig ${ }^{1}$ ) zeigte auf Grund von Experimenten, dass die Entdifferenzierung der Zellen bei Actinosphärium Eichhornii infolge von Ueberfütterung ausgelöst werde; dasselbe sei nach ihm auch bei Carcinom der Fall. So zeichnen sich in der Tat die Basalzellenkrebse, d. h. diejenigen Krebse, welche als in der nächsten Nähe der die Nahrung enthaltenden subepithelialen Schicht gelegen unter günstigen Ernährungsbedingungen sich befinden, durch besonders hohe Bösartigkeit von den Kankroiden aus (Krompecher, Der Basalzellenkrebs, 1903); sie haben ja ausserdem von vorneherein einen geringeren Grad der Differenzierung im Vergleich mit den mehr gegen die Oberfäche zu gelegenen Zellen. Bei Paralytikern kommt deshalb Krebs ${ }^{2}$ ) selten vor: Während Krebskranke meistens einen intakten Zirkulationsapparat haben, findet sich bei Paralytikern nahezu regelmässig eine meist vorgeschrittene Arteriosklerose mit Veränderungen am Herzen.

Also jede Reizung, Nahrungsmangel inbegriffen ${ }^{3}$ ) führt zur Vermehrung der Zellen. Dass dieser Reiz beim Carcinom nicht nur ein chronisch intermittierender ist, wie es die Kasuistik lehrt, sondern dass er es sein muss und dass es nicht anders sein kann, babe ich bereits bewiesen ${ }^{4}$ ), ebenso, dass die Zellvermebrung durch Zelluntergang eingeleitet wird. Nicht die Zellvermehrung ist das Primäre und ihr Untergang das Sekundäre, sondern der Tod der Zellen ist es, der zur Vermehrung der Zellen führt. Das geht aus meiner früheren Carcinomarbeit (Kap. II) ${ }^{5}$ ) und aus unserem Gesetz I hervor: je mehr Individuen zu Grunde gehen, um so mehr neue müssen entstehen. Sehr charakteristisch sowol in Bezug auf die Bedeutung des chronisch-intermittierenden Reizes, wie auf den der Vermehrung von Zellen vorausgehenden Untergang derselten sind die Nitteilungen Nalys ${ }^{6}$ ) über die Entstehung des Carcinoms von der nicht heilenden Erosion, indem, wie Maly so richtig hervorhebt, das neugebildete Gewebe jedesmal wieder vernichtet wird. Die Hinfälligkeit der Zellen bösartiger Ge-

1) Ueber physiologische Degeneration bei Aktinosphärium usw.

2) A. Fraeukel, Wiener klin. Wochenschr. 1905. No. 3.

3) Vergl. auch M. O. Wyss, Beitr. zur klin. Chir. Bd. XXIX. 1906.

4) Das Carcinom. Leipzig 1898, Kap. Il.

5) Weigert, Ziegler, Coen, Fischer, Nikiforoff, Obolonsky, Krafft, Podwyssodski kamen auf anderem Wege zu derselben Ansicht.

6) Centralbl. für Gynäkol. 1903. No.27. - Vergl. auch meine Ausführung in der Abhandlung "Das Carcinom". S. 6. 
schwülste ist fast ebenso gross, wie ihre Wucherungsfahigkeit. Das sieht man auch aus den Experimenten von Ribbert und Lubarsch, welche zeigten, dass es nur dann zu bedeutender Wucherung der implantierten epithelialen Gewebe kommt, wenn wenigstens Teile der epithelialen Gewebe zu Grunde gegangen waren ${ }^{1}$ ). In Lubarschs Versuch sah man die grossartigsten Wucherungen bei Implantation von Speicheldrüsenteilen eintreten, wo stets der grösste Teil des implantierten Gewebes zu Grunde geht, während nach Verlagerung von Lreteren- oder Nierenbeckenschleimhaut, ron Nebenhoden oder Knorpel - so gut wie keine Neubildung epithelialer Anteile beobachtet wurde, weil hier rom implantierten Gewebe nichts zu Grunde geht. Das beweist übrigens, wie richtig Lubarsch hervorhebt, dass die Ursache der Gewebsneubildung gar nicht in der Gewebsverlagerung, wie Ribbert meint, sondern in der Gewebsnekrose liegt. Deswegen sehen wir oft ein rapides Wachstum des Carcinoms nach Probeexzision, infolge ron Beleidigung von Warzen, Hauthörnern und kleinen Verwundungen (z. B. an der Zunge), nach der früher so oft geübten Palliativoperation des Uteruskrebses. So sehen wir auch, mit wie grosser Energie das Corpus luteum den Eiausfall zu ersetzen versucht, wie hier ein ziemlich geringes Trauma eine kolossale Zellwucherung zur Folge hat. Die Entstehung nach anderen Reizwirkungen ist ja allgemein bekannt, so dass ich auf diese Reizkategorien nicht näher einzugehen brauche ${ }^{2}$ ).

Wir sehen also, dass der Zellvermehrung eine Läsion vorausgeht und dass diese Läsion auch durch Nahrungsüberfluss bzw. Nahrungsmangel angefacht werden kann.

Ausser dem Nahrungsüberfluss gibt es, wie ich a. a. O. gezeigt habe, noch andere Momente, die eine Zellvermehrung hervorrufen. Vor allem Temperatur, Wasser, gewisse osmotische Bedingungen. $\mathrm{Zu}$ dem be-

1) Vergl. auch S. 607: „ohne Nekrose ist keine entzündliche Hyperämie möglich" (Ritter).

2) cf. das Carcinom, Kap. II. - Nach jedem Substanzverlust, z. B. an der Haut tritt rermehrte Zellenproduktion auf, eine wahre Luxusprodultion, die durch das hyperplastische Granulationsgewebe, durch die byperplastische Bindegewebsnarbe und durch den luxuriirenden Kallus demonstrirt wird. Nur tritt hier später Resorption ein, weil der Reiz und mit ihm die Nahrungszufuhr allmählich nachlassen, widrigenfalls müsste endlich daraus eine zelluläre Bildung entstehen. Regeneration ist nur eine Exageration eines normalen Prozesses, denn schon im physiologischen Zustande sind ja die Organe und die Gewebe in steter Erneuerung begriffen, indem jede Zelle durch eine neue ersetzt wird. Dieser unter gewissen Umständen mehr intensiv auftretende normale Prozess wird dann evidenter. Da das Leben nur eine Reihe fortwäbrender Regenerationsvorgänge darbietet, so wäre eher die Frage: "warum gewisse Elemente sich nicht regenerieren?" richtiger, als die Frage: "warum tritt Regeneration auf?" 
reits in meiner rorigen Arbeit $\left.{ }^{1}\right)$ Gesagten will ich noch folgendes hinzufügen: Aus den Versuchen von Loeb u. A. ${ }^{2}$ ) geht hervor, dass (z. B. bei Tubularien) eine Beschränkung der Wasseraufnahme eine Verzögerung der Degeneration bis zur vollständigen Hemmung hervorruft; umgekehrt beschleunigt das Hinzufügen von $\mathrm{H}_{2} \mathrm{O}$ zum Seewasser die Regeneration, bei zu starker Verdünnung tritt wieder eine Verzögerung der Regeneration ein. Die Wasseraufnabme wird, nach Loeb, durch den Unterschied des osmotischen Druckes im Organismus und in der umgebenden Lösung bestimmt. Es lässt sich somit das Wachstum durch Aenderung des osmotischen Druckes beeinflussen. Herbst ${ }^{3}$ ) zeigte, dass man durch willkürliche Aenderung der chemischen Zusammensetzung des Meereswassers auch die Entwickelung der Seeigellarven in andere Bahnen lenken kamn und dass diese Veränderung nicht auf einer chemischen Wirkung der zugeführten Stoffe beruht, sondern dass sie auf die veränderte physikalische Eigenschaft, speziell auf den veränderten osmotischen Druck des umgebenden Mediums zurückzuführen ist: Er stellte auch fest, dass die Wirkungstärke der Salze der Halogengruppe auf den wachsenden Organismus vom Chlorid bis zum Jodid, also mit der Steigerung des Molekulargewichtes, abnimmt. Die Höbe der Beeinflussung des Wachstums ist daher speziell abhängig von der Auzahl der Salzmoleküle, welche in demselben Volum enthalten sind. Nach C. Ritter (Mitteil. aus den Grenzgebieten der Medizin und der Chirurgie. XIV. 3. S. 235. 1905) „bedingt die Schädigung des Gewebes eine Erhöhung des normalen osmotischen Druckes in den Geweben und ihren Flüssigkeiten, und so muss nach rein physikalischen Gesetzen das Blut angelockt werden. Ohne Nekrose ist keine entzündliche Hyperämie möglich. Wenn durch den Eiweisszerfall die Gewebssäfte in höhere osmotische Spannung versetzt werden, so muss nach dieser konzentrierten $\mathrm{Ge}$ websflüssigkeit hin ein Flüssigkeitsstrom von allen den Orten her stattfinden, an denen ein geringerer osmotischer Druck berrscht. Das ist aber in allen Blut- und Lymphkapillaren der Fall, die ja den osmotischen Druck

1) Das Carcinom. Kap. III.

2) Loeb, Organbildung und Wachstum. Würzburg 1892. - Arch. f. Entwickelungsmechanik. 1898. S. 631-642. - Ueber den Einfluss von Alkalien und Säuren auf die embryonale Entwicklung und das Wachstum. - Cf. auch Herbst, Mitteil. der zoolog. Station zu Neapel. 1893. S. 136. Arch. f. Entwickelungsmechanik. 1896. S. 455, 470, 482, 497, 503. - Hertwig, Beitr. zur experim. Morphol. und Entwickelungsgeschichte. 1895. 44. S. 283; Butaillen, Schimkewitsch, Derjugin, Gurwitsch, Arch. f. Entwickelungsmechanik. 1896. 3. S. 49. - Wils on, Ibid. 1897. 5. S. 617. - Davenport, The role of water in Growth. Proceedings of the Boston Society of Natural History. 1897. Vol. 28. p. 53,84 .

3) Zeitschr. f. wissenschaftl. Zoologie. Bd. XXV. S. 446-516. 
der normalen Gewebssäfte haben. Sie umspannen ja die Zellen bzw. die Zellkomplexe unmittelbar und sind auch von den Gewebssäften durch eine Membran getrennt."

Zwei andere physikalische Bedingungen des Wachstums sind: Eine gewisse Turgeszenz der Zelle und Ausdehnungsfähigkeit der protoplasmatischen Hülle ${ }^{1}$.

Das Verhältnis des Wassers zur festen Masse des Körpers wird mit den Jahren geringer. Dementsprechend nimmt die Empfindlichkeit gegen Wasserentziehung bei Embryonen mit fortschreitendem Alter $a b\left(\mathrm{Loeb}^{2}\right)$ ). Drei Perioden sind hier zu unterscheiden (Davenport): Zu Anfang der Entwickelung findet sich eine Periode sehr rascher Zellteilung, aber geringer Volumszunahme; es folgt dann die Periode reichlicher Wasseraufnahme, die von entsprechendem Wachstum und von der Ausbildung der Körperform nebst Anlage der einzelnen Organe begleitet ist, während eine dritte Periode die Differenzierung der Gewebe unter Herabminderung des Wachstums besorgt.

Wie gross die Rolle der Wasserzufuhr bei Carcinom ist, erhellt daraus, dass gerade ein wichtiges Kennzeichen bösartiger Geschwülste in ihrer abnormen Weichheit - einer Eigenschaft aller niederen Organismen und in ibrem abnormen Flüssigkeitsreichtum liegt (Krebssaft, medullare Tumoren, weiche, extrem rasch wachsende Sarkome [Kahane $\left.\left.{ }^{3}\right)\right]$ ). Dem Mammacarcinom geht oft Ausfluss von serösem Exsudat voraus. Ein Skirrhus steht an Bösartigkeit weit hinter dem Medullarkrebs zurück, ein derbes Fibrosarkom besitzt eine geringere Malignität als ein gallertartig weiches Rundzellensarkom oder als ein halbflüssiges Sarkom. Der Satz: je weicher und saftreicher eine maligne Geschwulst, desto grösser auch ibre Bösartigkeit, hat also seine volle Geltung - die Konsistenz eines malignen Tumors gewährt wertvolle Aufschlüsse über die Prognose, auch quoad Rezidive; im grossen Ganzen steht die Bösartigkeit einer Geschwulst zu der Härte ihrer Konsistenz in einem umgekehrt proportionalen Verhältnis. Die spezialisierten Zellen - sagt $\left.\mathrm{Fol}^{4}\right)$ - sind eines fast unbeschränkten Wachstums fähig, sie können sich aber nicht oder nur in sehr beschränktem Masse vermehren, wenn sie nicht zuvor durch Verflüssigung der differenzierten Teile in den embryonalen Zustand zurückgefallen sind. (Vergl. Ss. 613, 615.)

Was den Einfluss von Sauerstoff anbelangt, so ist bekaunt, dass bei

1) Hemmeter, The Americ. Journ. of the med. sciences, 1903. Vol. XXV. New Series. p. 687.

2) Pflügers Archiv. 1894. Bd. 55. S. 530.

3) Centralbl. f. Pathologie. 1895.

4) Lehrb. d. vergl. Anat. S. $315 \mathrm{ff}$. 
Verminderung der O-Aufnahme die Embryonalentwicklung eine Verlangsamung erleidet ${ }^{1}$ ). Im Gegenșatz zu Temperatur und Wasser nimmt die Empfindlichkeit gegen Sauerstoffentziehung mit fortschreitender Entwicklung zu. Gelingt es, Substanzen in den embryonalen Organismus einzuführen, welche eine Beschleunigung in der Aktivierung des Sauerstoffs und eine Zunahme der Oxydationsvorgänge und damit des synthetischen Prozesses ermöglichen, so muss eine Beschleunigung der Entwicklung und des Wachstums stattfinden. Eine derartige beschleunigende Oxydationswirkung besitzen die Alkalien; der zur normalen Entwicklung notwendige Grad von Alkalinität des Nediums wird im Wesentlichen von den Bikarbonaten geliefert. Säuren haben dagegen nur einen hemmenden Einfluss auf das Wachstum und die Entwicklung. Verschiedenheiten in Alkali- und Säuregehalt verschiedener Stellen des Keimes könnten [Loeb, Davenport, Herbstat)] sehr wohl einer der Umstände sein, welche die zur Differenzierung des Embryos nötigen Wachstumsungleichheiten herbeiführen. Sie sind aber natürlich nicht die einzigen Verschiedenheiten.

Ueberhaupt kann man durch Zusatz von gewissen Körpern die Entwicklung hemmen oder beschleunigen ${ }^{3}$ ). So verläuft die Entwicklung der Eier von Echiniden gar nicht oder doch nicht vollständig, wenn dem Meerwasser eines der Elemente $\mathrm{Na}, \mathrm{K}, \mathrm{Cl}, \mathrm{S}, \mathrm{Mg}$, $\mathrm{Ca}$, vielleicht auch $\mathrm{Fe}$ fehlt - sie sind ebenso wie ein gewisser Grad von Alkalinität des Mediums für die Entwicklung notwendig ${ }^{4}$ ). Setzt man statt $\mathrm{SO}_{4} \mathrm{SO}_{3} \mathrm{zu}$, so tritt Verlangsamung des Wachstums ein, da $\mathrm{SO}_{3}$ den Sauerstoff an sich reisst, um sich $\mathrm{zu} \mathrm{SO}_{3} \mathrm{OH}_{2}$ zu vervollständigen ${ }^{5}$ ).

1) Godlewski, Arch. f. Entw.-Mech. 1901. Xl. S. 585. - Loeb, Pflügers Arch. 1895. Bd. 62. S. 24. - Cf, auch Roux, Hallez, Samassa.

2) Herbst, Arch. f. Entw.-Mech. 1897. S. 649 und 1898. S. 480. Bd. VII.

3) Herbst, Hertwig, Gurwitsch, Wilson.

4) Diese Körper können durch andere nur in ziemlich engen Grenzen ersetzt werden: $\mathrm{K}$ durch $\mathrm{Rb}$ oder $\mathrm{Cs}, \mathrm{SO}_{4}$ durch $\mathrm{S}_{2} \mathrm{O}_{3}$, Cl durch $\mathrm{Br}-$ Befunde, die sich mit den von der botanischen Ernährungsphysiologie ermittelten ungefähr decken.

5) Herbst (Arch. f. Entw.-Mech. 1900. S. 424) gibt an, dass Ca nötig ist, um Furchungszellen der Echiniden in Verbande zu halten; im Ca-freien Wasser fallen die Blastomeren des (membranlos gemachten) Seeigelkeimes auseinander: anstelle einer Blastula erhalten wir gegen 1000 einzelne wimpernde Zellen. Herbst erzielte durch Zusatz gewisser Salze, z. B. der Li-Salze zu dem Seewasser, in welchem Seeigeleier sich entwickelten, eine recht auffallende und typisch wiederkehrende Abweichung in der Gestalt der Larven („Lithiumlarven“). Aehnliche Modifikation erzielte Driesch durch erhöhte Wärmezufuhr, Hertwig durch Aenderung der Konzentration. Bonnier erhielt bei Ononis natrix verschiedene morphologische und histologische Differenzen, je nach dem Boden, ob Ca-haltig oder Ca-frei. 
Dass das Licht einen grossen Einfiuss auf die Entwicklung der Zellen hat, das ist uns bereits aus der Pfanzenwelt bekannt. Artur Smith hat gezeigt, dass Pflanzen, welche während der Nacht durch elektrisches Licht beleuchtet werden, rasch wachsen, aber meistens vorzeitig zugrunde gehen. Im grossen Ganzen wirkt aber Dunkelheit beschleunigend auf das Wachstum der Pflanzen ein, während Beleuchtung dasselbe verlangsamt. Pflanzen wachsen also im allgemeinen bei Nacht stärker als bei Tage. Bei Thuja occidentalis kann man, durch Umdrehung der Zweige, erreichen, dass die Zellen, welche sonst die charakteristische Gestalt der Zellen der Unterseite angenommeu hätten, jene Gestalt erlangen, welche unter normalen Verhältnissen den Zellen der stärker belichteten Oberseite zugekommen wäre. Verschiedene Lichtstrahlen üben einen verschiedenen Einfluss auf das Wachstum der Zellen aus ${ }^{3}$ ).

Was den Einfluss von Temperatur anbelangt, so wissen wir, dass eine in gewissen Grenzen erhöhte Temperatur das Wachstum befördert. Es ist auf Grund der Versuche von Gallow ay ${ }^{4}$ ) nicht unwahrseheinlich, dass die Wirkung der Temperatur keine direkte sei, sie beeinflusse nicht das eigentliche Wachsen organischer Substanzen; ihr Einfluss sei vielmehr ein indirekter, indem die erhöhte Temperatur die Geschwindigkeit der Wasseraufuahme befördere. Licht und Temperatur können sich manchmal gegenseitig ersetzen: das Dunkler- oder Hellerwerden mancher Larven, z. B. beim Salamander, unter Einfluss von Licht kann auch durch Temperaturerhöhung erzielt werden ${ }^{5}$ ). Vermehrung der Wärmezufuhr wirkt in derselben Weise wie Nahrungsüberfluss: bei Yögeln und Insekten z. B. kann man die Reifung der Eier beschleunigen oder verlangsamen, je nachdem wir die Temperatur erhöhen oder erniedrigen. Ilaupas ${ }^{6}$ ) hat nachgewiesen, dass mit steigender Temperatur, bis zu einer gewissen Grenze, die Zahl der in 24 Stunden erfolgenden Teilungen der Infusorien (an 20 Infusorienarten geprüf) proportional zunimmt. Es ist bekannt, dass die Embryonalentwicklung der eierlegenden Tiere bei erhöltter Temperatur (wie z. B. auch in tropischen Gegenden) schneller verläuft als im Norden oder bei gewöhulicher Bruttemperatur. Ebenso kann bei Planarien durch Temperaturerhöhung die Regeneration eines ganzen Tieres beschleunigt werden und zwar von 12-15 Tagen normaler Dauer bis auf 4-5 Tage.

Mit fortschreitender Entwicklung nimmt sowohl die schädigende wie

1) Siehe James Nevins Hyde, On the influence of light in the production of cancer. The Amer. journ. of the med. sciences. 1906. No. 1.

2) Study on the cause of acceler. effect of heat upon growth. Amer. Natur. 1900. 34. p. 949.

3) Osk. Hertwig, Die Zelle und die Gewebe. Bd. II. S. 117.

4) Archiv. de zool. expér. et génér. Sér. XII. T. 6. 1888. 
die fördernde Wirkungskraft von Temperatur, ähnlich der Empfindlichkeit gegen Wasserentziehung ab; zur Zeit der Embryonalentwicklung ist sie am grössten (Verworn). Unterbalb einer bestimmten Temperaturgrenze werden die Entwicklungsrorgänge sistiert, obne dass darum der Tod sofort einzutreten pflegt. Die Abhängigkeit der Geschwindigkeit der Formbildungsprozesse von der Wärmezuluhr bietet grosse Analogien zu den Phänomenen der chemischen Reaktionen ${ }^{1}$ ).

Vielleicht wirkt die Temperaturerhöhung bei entzündlichen Krankheiten günstig nicht durch Tötung der Bakterien, sondern durch energische Vermehrung der Zellen des Organismus.

Ad 3. Wir haben gesehen, dass durch grössere Nahrungszufuhr die Zelleu rascher eine zu grosse Masse im Verhältnis zur Oberfläche erlangen, so dass sie sich dann früher teilen als normaliter, $d . h$. die Teilungen folgen dann schneller, häufiger aufeinander. Die Nahrungszufuhr zu den Carcinomzellen wird immer grösser und dadurch kommt es zu einem immer schnelleren Rhythmus in der Teilung der Zellen ${ }^{2}$. Alles, was sich auf einem jüngeren Stadium vermehrt, vermehrt sich auch häufiger — das ist ein allgemeines Naturgesetz. Aber eine früb, d. h. auf einer jüngeren Entwickelungsstufe sich teilende Zelle lässt Zellen hervorgehen, die erstens die erblich erworbene Neigung besitzen, sich ebenfalls früh, d. b. anf einer niedrigeren Entwickelungsstufe fortzupflanzen; zweitens werden diese Zellen dadurch ein kleineres Volumen besitzen und als kleine Zellen vermehren sie sich stärker als die grossen. Starke Vermehrung ist ja die Eigenschaft kleinerer, niedriger organisierter Wesen: so sterben manche Insekte (Fliegen, Bienen, Schmetterlinge) bald nach der Paarung, als Zeichen, dass ihr Lebenszweck eben in der Vermehrung liegt.

In der Tat entsprechen die Verhältnisse beim Carcinom diesen Gesetzen. Die Zellen des Carcinoms werden mit der Zeit immer kleiner und kleiner und sie erwerben mit der Zeit eine immer stärkere Propagationsfähigkeit, i. e. Bösartigkeit. Auch nimmt die spezifische Funktion der

1) Peter (Physik.-med. Gesellsch. in Würzburg, Sitzung rom 20. VII. 1905) fand für Seeigeleier, dass zwischen $2,5^{\circ}$ und $25,0^{\circ}$ für jede $10^{\circ}$ die Schnelligkeit der Entwicklung fast um das $21 / 2$ fache zunimmt. Dieses Resultat stimmt mit dem van t'Hoffschen Gesetz, nach welchem bei weitem die meisten Reaktionen beim Ansteigen der Temperatur um $10^{\circ}$ Verdoppelung bis Verdreifachung der Geschwindigkeit aufweisen und spricht für Hertwigs Ansicht, dass ein grosser Teil der "Entwicklungsarbeit" chemische Arbeit ist. Bei steigender Temperatur nimmt das Geschwindigkeitsverhältnis für $10^{\circ} \mathrm{ab}$, ebenfalls in Uebereinstimmung mit dem ron van t'Hoff für chemische Reaktionen aufgedeckten Gesetz.

Erhöhte T'emperatur oder mit anderen Worten vergrösserte molekulare Vibration beschleunigt auch die Diffusion der Kristalloide.

2) Cf. mein "Carcinom", Kap. III. 
Zellenart, aus der das Carcinom hervorgegangen, proportional ab, indem die allgemeinen Funktionen, d. h. die der Vermehrung; zunehmen ${ }^{1}$ ). (Dass die allgemeinen Funktionen der Zellen eben in Vermehrung bestehen, sieht man daraus, dass bei denjenigen Insekten, welche bald nach der Parung sterben, diejenigen Exemplare, welche nicht zur Paarung gelangt sind, bis zum nächsten Frülijahr am Leben bleiben.) Da auf diese Weise die Zellen des Carcinoms sich auf einer niedrigeren Entwickelungsstufe teilen und die dabei resultierenden abortiven Tochterzellen die Eigenschaften der unvollständig entwickelten, jungen Mutterzellen erben ${ }^{2}$ ), so ist das in gewissem Sinne eine Erscheinung der Neotenie ${ }^{3}$ ), ein Ausdruck, der bereits für ganze Organismen angewendet wird und aus dem Grunde, dass er nicht nur den Rückgang bezeichnet, sondern auch die Entstehung desselben mit-

1) Es sollen bei somatischen Zellen (sowohl bei Pflanzen wie bei Tieren) die Chromosomen (bei der Zeliteilung) in longitudinaler, reproduktive in transversaler Richtung sich teilen, wobei die reproduktiven Zellen nur ungefähr halb so viel Chromosomen bilden und die Eigenschaft haben, somatische Zellen in ibrer Umgebung zu zerstören (z. B. im Embryonalsack der Pflanzen). "Aehnliche Zellteilungen wie bei reproduktiven Zellen sind auch in einigen Zellen aller untersuchten malignen, nie bei benignen Neubildungen $z u$ finden. Bei Pflanzen seien Umwandlungen somatischer Zellen in reproduktive bekannt." (Lancet 1904. 6. II, Former und 13. III, Bashford and Murray).

2) Dans les parties où l'organisation du blastème se fait très-vite, les éléments n'arrivent pas au dernier stade de leur développement (B o ca. Les tumeurs. p. 18i). Cf. auch Jaekel, O., Ueber verschiedene Wege phylogenetischer Entwickelung. Verh. des V. internation. zoolog. Kongresses zu Berlin 1901 (Jena 1902) and The Erasmus Wilson Lectures on the general pathology of tumours by Charles Powell White.

3) Kollmann und Boas haben uns vielfache Beispiele von Neotenie mitgeteilt. Es vermehren sich z. B. einige Fische noch in unreifem Alter; aber die Nachkommenschaft solcher nicht vollständig entwickelten Tiere erlangt nie das normale Volumen ihrer Spezies, sie erlangt nur die Grösse ihrer unmittelbaren Eltern. Mit einem Wort, nicht vollständig entwickelte, auf einer niedrigeren Entwickelungsstufe stehen gebliebene Formen können nur eine Nachkommenschaft von geringem Volumen erzeugen. So werden bei jungen Eltern atavistische Eigenschaften besonders stark hervortreten (Mehnert, Biomechanik. S. 158); das sehen wir auch in den Krebszellen. - Viele Evertebraten werden schon in der Jugend proliferationsfähig, manche besonders frühzeitig. So werden Ctenophorenlarven geschlechtsreif nach Verlassen der Eihülle und legen befruchtete Eier ab. Selbst noch unter den Tunikaten kann eine ungeschlechtliche Vermehrung bisweilen schon während des Larvenlebens erfolgen. Das Gegenteil zeigen die Repräsentanten der höheren Tierklassen: sowohl bei Vögeln, als auch beim Menschen beginnt die Generationsperiode erst dann, wenn die Tiere im grossen und ganzen ausgewachsen sind (Mehnert, Biomechanik. S. 159). Viele Amphibien der Jetztzeit stellen z. T. nur neotenisch stark degenerierte Formen dar. 
fasst, richtiger ist, als Anaplasie oder Katabolismus, welche nur die Rückbildung, nicht aber den Weg, auf welchem diese entsteht, bezeichnen. Von noch allgemeinerem Standpunkt aus betrachtet, wäre dieser Prozess - da die Carcinomzellen allmählich nicht nur die anatomischen, sondern auch die physiologischen Eigenschaften niedriger organisierter Zellen annehmen (d. h. sie erlangen immer weniger spezialisierte und mehr allgemeine Eigenschaften) - als Entdifferenzierung - und da die Carcinomzellen, als niedere Zellen, immer mehr und mehr von anderen Körperelementen unab̆ängig werden - zugleich als Desintegration im Sinue Spencer's zu bezeichnen'). Das alles erklärt uns zur Genüge die Ursache der massenhaften Zellvermehrung bei Carcinom.

Stellt die Anaplasie etwas Besonderes, eine nur für das Carcinom charakteristische Erscheinung vor? Schon von vornherein würden wir, wenn wir uns erinnern, dass, wie wir das schon seit Bichat und Laënnec2) und nicht nur seit Virchow, wie allgemein irrtümlich angenommen wird, wissen, die Tumoren überhaupt nichts dem Körper absolut Fremdes vorstellen, da sie ihre Vorbilder in anderen, auch normalen Vorgängen haben - zu dem Schluss kommen müssen, dass auch die Anaplasie keine exklusiv für Carcinom charakteristische Erscheinung ist. Auf den ersten Blick ist es etwas schwer zu verstehen, wie sich überhaupt die Zellen und ihre Eigenschaften ändern können, z. B. im Laufe der Embryonalentwickelung. Allein es gibt ausser Carcinom viele andere pathologische, aber auch normale Prozesse, wo ebenfalls anaplastische Prozesse vorkommen. Schon in meiner früberen Arbeit ${ }^{3}$ ) habe ich darauf hingewiesen, dass es a) keine direkte zelluläre Variation eines Lipoms gibt und dass, wenn ein Lipom in Sarkom übergeht, es sich zuerst in die em-

1) Bereits in den 70er Jahren des vorigen Jahrhunderts baben Heitzman n und Stricker über diese Entdifferenzierung der Zellen geschrieben. - Auch die Form der Wucherung beim Carcinom erinnert an embryonale Prozesse, so das Wachstum nach innen zu. Beneke (Roux' Arch. 1899. S. 695, Dtsch, Arch. f: klin. Med. 1899. Bd. 64. S. 227--265) beschrieb einen merkwürdigen Befund von Carcinom in dem mit mässigem Quantum von Transsudat gefüllten Pleurasack, wo sich Blasen fanden, die aus einer 10-15 schichtigen Krebszellenlage bestanden, welche ihrerseits mit Flüssigkeit gefüllt waren, in der einige Zelltrümmer und Leukozyten schwammen. Dieses Lumen war nicht durch Nekrose oder Auflösung zentral gelegener Zellen entstanden, sondern es handelte sich. nur um eine spezifische Wachstumsform der Epitelzellen, d. i. eines Drüsenlumens in Gestalt einer Kugelblase. Die Bildungen glichen in bohem Masse dem Blastulastadium des Embryo.

2) Broca, 1. c.

3) S. $18 \mathrm{ff}$. 
bryonale Vorstufe des Fettgewebes, in das Schleimgewebe (cf. Ss. 608 u. 615) umwandelt, um danu als Myxosarkom zu erscheinen ${ }^{1}$ ). b) Carcinome mit Flimmerzellen kommen nicht vor. Wenn Carcinome in mit Flimmerzellen bekleideten Organen vorkommen, z. B. in den Atmungswegen, in den Tuben, im Uterus, so verlieren die Zellen dabei ibren Flimmerbesatz, der als Zeichen höherer Differenzierung gilt ${ }^{2}$ ). c) Auch das Periost geht nach Knochenfraktur auf eine frübere Entwickelungsstufe zurück: seine Elemente werden indifferenter (Ribbert $\left.{ }^{3}\right]$ ), indem sie dann Mark, Knorpel, Knochen und auch normales Periost liefern, als Reminiszenz an frübere Zeit, wo das Periost noch Perichondrium war; durch diese Vorgänge wird in modifizierter Weise die Embryonalentwickelung des Knochensystems wiederholt. Dieselbe Eigenschaft weist Ribbert auch für andere Gewebe nach ${ }^{4}$ ). Leberhaupt existiert zwischen denjenigen Zellen, die Neigung zur Wucherung besitzen, eine Verwandschaft, wie schon Virchow ${ }^{5}$ ) angegeben, z. B. zwischen Schleim- und Knorpelgewebe. Das erklärt sich

1) Borst sagt, es sei ihm diese, ihm nur aus dem Lebrbuch von $R$. Thoma bekannte Tatsache bis jetzt nicht vorgekommen, er spricht davon mit einem gewissen Skeptizismus. Ich kann hier nur hinzufügen, was bereits Virchow in seiner Geschwulstlehre sagt (S. 405 ff.) „Selbst das Fettgewebe wuchert nicht als solches, sondern es wird, wie das Knochen- und Knorpelgewebe, in der Regel erst in ein weiches, mehr schleimiges Gewebe transformiert und, genau genommen, ist es dieses, welches proliferiert. ${ }^{~}$ - Schleimgewebe kann sich aus allen wuchernden Bindesubstanzen entwickeln, es ist meist ein transitorisches Gewebe, das in Fettoder Bindegewebe übergeht (Ziegler, Lehrb. d. Pathol. I. S. 312. VIII. Aufl.). Dass typische Fettzellen sich wieder in sternförmige (Schleim-) Zellen umwandeln und dass die letzteren sich unter Umständen wieder zu Fettzellen entwickeln, hat auch Kölliker (Arch. f. mikrosk. Anatomie. II. 1. S. 19. Fig. 92) angegeben. Ueberhaupt vermehren sich die spezialisierten Zellen nur dann stark, wenn sie zuvor durch Verflüssigung der differenzierten Teile in den embryonalen Zustand zurïckfallen ( $\mathrm{Fol})$. - Nicht nur anatomisch stellen die Schleimzellen eine niedrigere Stufe vor. Denn das Muzin stellt auch in chemischer Hinsicht eine minderwertige Substanz im Vergleich mit den echten Eiweisstoffen vor, indem es einen weit geringeren Gebalt an Kohlenstoff aufweist (Mühlmann). Myxom entsteht gewöhnlich im Fettgewebe.

2) v. Hansemann, S. 34. - Es vermehren sich nicht im reifen Organismus Ganglien-, Sinnes- und Flimmerzellen. (Gurwitsch, Morphologie und Biologie der Zelle. Jena. 1904.)

3) Ueber Rückbildungen an Zellen und Geweben (S. 9).

4) Allerdings wird man nicht erwarten dürfen, dass ein Gebilde genau zu seiner Anfangsform zurückkehrt, weil die vielen, dasselbe während seiner rückläufigen Entwickelung treffenden Einflüsse die verschiedensten Ablenkungen bedingen können (Jickeli, S. 279).

5) Geschwülste. 
durch die Embryogenese des Knorpels, welcher aus dem embryonalen Gallertgewebe entsteht ${ }^{1}$ ).

d) Ribbert ${ }^{2}$ ) fand, dass bei ganz kleinen, in Lymphdrüsen oder in die Bauchhöhle transplantierten Stückchen der verchiedenen Organe und Gewebe die Zellen nicht zugrunde gehen, vielmehr eine eigentümliche Rückbildung auf eine frühere Entwickelungsstufe (Entdifferenzierung) durchmachen und dann lange (Monate bis über ein Jahr lang) erhalten bleiben. e) Auch durch Atrophie entstehen Entdifferenzierungen. So werden die Epithelien der gewundenen Harnkanälchen bei atrophischen Vorgängen insofern indifferent, als sie oft gar nicht mehr von denen der geraden Kanälchen zu unterscheiden sind; atrophische Hodenepithelien gleichen völlig den Nebenhodenepithelien oder sind ihnen sehr ähnlich, ebenso wie atrophische Fettzellen gewissen Bindegewebs- oder Sternzellen ähnlich oder gleich sehen ${ }^{3}$ ).

Aebnliche Entdifferenzirung stellt auch die f) Narbe vor, indem sie nicht wieder die ursprüngliche Form herstellt. Es sind die Granulationen in den Verbrennungsnarben oft sehr schwierig von einem geschwulstartigen Gebilde zu unterscheiden, meint Virchow; der Unterschied ist nur durch die Dauerhaftigkeit zu bestimmen. Hierher gehören auch die Naevi ${ }^{4}$ ). Die Warze, sagt Kronthal ${ }^{5}$ ), aus der sich oft maligne Geschwülste entwickeln, stellt in ihrer einfachsten Form, ganz abgesehen von den fibroiden, melanotischen und anderen Formen, schon ein Gebilde dar, in welchem zellige Elemente gegeneinander in anormaler Weise orientirt sind. Und auch g) die Regeneration gewisser Körperteile, wie z. B. des Schwanzes bei den Eidechsen oder der Augen bei den Schnecken, des ganzen Verdauungskanals bei Holothurien erinnert an dieselbe Entdifferenzierung, indem jedesmal das neurestaurierte Glied in einer weniger vollkommenen Form erscheint. Auch ohnedies bedeutet ja jede Regeneration einen embryonalen Prozess. Denn in allen Fällen, in denen ein Organ oder ein Gewebe fähig ist, sich wieder zu erzeugen, muss es Elemente von embryonalem Charakter enthalten $\left.{ }^{6}\right)$.

1) Die Sternzellen in der Neuroglia äbneln den Knorpelzellen; ihnen noch ähnlicher ist das Schleimgewebe.

2) Arch. f. Entwickel.-Mechanik. VI. Heft 1.

3) Lubarsch, S. 258.

4) Cfr. Carcinom S. 19, 20.

5) 1. c. S. 248.

6) Daraus, dass am atgeschnittenen Ende nur die verloren gegangenen Teile und nicht z. B. Schwanz an Stelle von Kiemen regeneriert wird, muss man den Schluss ziehen, dass die alten Zellen jedesmal neue Zellen nur der nächsten Reihe erzeugen, aus der wieder eine Reihe nächst differenzierter Zellen entstehen, ähnlich wie z. B. bei normaler Regeneration der Haut. 
Und wir sehen auch, dass je tiefer ein Tier in der phylogenetischen Reibe steht, um so vollkommener die Regeneration ist. G. Messing ${ }^{1}$ ) hat experimentell nachgewiesen, dass je tiefer entwickelungsgeschichtlich das Tier steht, umsomehr die für die typische Entzündung (nach Durchziehen von Fäden bei Vollusken, Würmern und Cölenteraten) charakteristischen Erscheinungen verschwinden und an deren Stelle Regenerationprozesse auftreten. Aber schon bei einfacher h) Entzündung haben wir Erscheinungen der Anaplasie, der Rückkehr zu mehr ursprünglichen Verhältnissen. Wenn z. B. fibrilläres Bindegewebe in Entzündung gerät, so verschwinden die Fibrillen, die Zellen werden protoplasmareicher, wir sagen: das Bindegewebe wird zellig. Die Zellen können sich dabei von ihrer Lmgebung loslösen und sogar aktive Bewegungen ausführen ( $\nabla$. Hansemann)2). Schon bei gewöhnlichen Wucherungsprozessen nehmen die Endothelien als Bindegewebszellen nicht selten einen ganz epithelialen Charakter an - sie.werden entdifferenziert. Nicht nur dass sie kubisch oder gar ganz zylindrisch werden, sondern sie gehen auch epitheliale Verbände ein, ohne eine Interzellularsubstanz zu bilden und machen es schliesslich unmöglich, sie vom Oberflächenepithel zu unterscheiden (v. Hausemann, S. 50).

i) Nach Kölliker ${ }^{3}$ ) gibt es noch im erwachsenen Organismus Zellen mit embryonalem Charakter, nämlich:

1. Alle tieferen Zellen der geschichteten Epithelien und des Horngewebes, wie z. B. die Zellen des Haarknopfes, das Linsenkapselepithel, die tiefsten Zellen des Rete Malpighii der Epidermis ${ }^{4}$ ).

2. Die Osteo- und Odontoblasten.

3. Viele Knorpelzellen ${ }^{5}$ ).

1) Centralbl. f. allg. Path. und path. Anatomie. XIV. 22. S. 915. 1903.

2) Geschwülste. I. Aufl. S. 24.

3) Erinnerungen. S. 358.

4) Dementsprechend weist auch der Basalzellenkrebs (der aus den wenig differenzierten kleinen [chromatischen] Basalzellen der Oberflächenepithelien besteht) die grösste Malignität, d. h. die stärkste Wucherungsfähigkeit ron allen Hautkrebsen (Krompecher, Der Basalzellenkrebs, 1902) auf.

5) Daraus erklärt es sich, dass auch reine Chondrome, indem die Knorpelzellen schon normal einen embryonalen Charakter besitzen, Metastasen machen können, wäbrend das z. B. bei reinen Osteomen nie der Fall ist (Hansemann, I. Aufl. S. 46). - Es gibt bekanntlich $z$ wei Arten von Knorpeln, deren Unterschied nicht durch ihre Strultur, sondern durch ihre Lebensgeschichte hervortritt, nämlich den sogenannten temporären und den permanenten Knorpel. Der permanente Knorpel verharrt während des ganzen Lebens im Zustande des Knorpelgewebes, der erstere geht früher oder später in Knochengewebe über. Den beiden Arten von Knorpel entsprechen zwei verschiedene Enchondrumarten, von denen das eine gut-, das andere bösartig ist. Der Knorpel, welcher verknöchert, 
4. Die Flemente aller Drüsen, die Zellen bilden.

5. Die lymphoiden Zellen.

6. Gewisse Bindesubstanzzellen.

7. Die Keimzellen (Eizellen und Samenfädenbildungszellen) ${ }^{1}$ ).

Bereits Boll betrachtete das Finstehen von Carcinom als eine Rückkehr der Zellen zum Keimgewebe infolge von Alteration der Blutgefässe ${ }^{2}$ ).

Auch k) die Schilddrüse liefert ein scbönes Beispiel der sozusagen normalen Anaplasie: sie entwickelt sich beim Menschen aus einer Anlage, die als rudimentäres Organ zu denken ist. Der Weg; auf dem sie entstebt - sagt Gegenbaur ${ }^{3}$ ) - erinnert an neoplastische Prozesse. Es ist ein Wachstum aus einem in gewissem Sinne anaplastisch gewordenen Gewebe.

Was die Massenhaftigkeit der Zellvermehrung betrifft, so haben wir ebenfalls Analogien in normalen Prozessen, so 1) im Corpus luteum ${ }^{4}$ ), in der Decidua - worauf schon Virchow aufmerksam gemacht hatte, und in den dem Corpus luteum zum Teil entsprechenden $Z$ wischenzellen des Hodens. - Auch das Ei liefert uns ein Bild einer massenhaften Zellvermehrung und stellt eigentlich einen Rückdifferenzierungsprozess vor, indem es nach einer Reihe von Veränderungen wieder zu derjenigen Form zurückkehrt, aus der es hervorgegangen ${ }^{5}$ ); erst später folgt eine Differenzierung. Es

zeigt eine reichliche Gefässentwickelung; während der andere fast gefässlos ist. Dieser Unterschied beider Knorpelarten stellt nach A I bert das Paradigma des Unterschiedes zwischen embryonalem Gewebe, (Fortschritt von einem einfachen za einem komplizierten Zustand) und Krebsgewebe (Fehlen von Veränderung in der Richtung der Weiterentwickelung) vor. Schon Virchow meinte, dass zwischen Schleim- und Knorpelgewebe wahrscheinlich eine Verwandtschaft existiere. Bekanntlich entstebt das Knorpelgewebe aus dem Gallertgewebe, und zwar dadurch, dass das embryonale Gallertgewebe an einzelnen Stellen durch Wucherung zellenreicher wird (cf. S. 614).

1) Die Vegetationspunkte einer Polypenkolonie sind nach Kölliker, ebenfalls wie bei Planzen, auf Zellen von embryonalem Charakter zurückzuführen.

2) Es hat schon Virchow auf die Aehnlichkeit des Lungenbaues mit Carcinom hingewiesen. Auch Boll (S. 23) vergleicht die Carcinomstructur mit der Lunge, die, ähnlich wie beim Carcinom, das Resultat eines komplizierten Durchwachsungsprozesses des Epithels mit dem Bindegewebe und des Bindegewebes mit dem Epithel darstellt.

3) Anatomie des Menschen.

4) Die morphologische Aehnlichkeit der Zellen des Corpus luteum, der Nierenund Knochengeschwulstzellen hat Lubarsch (S. 272) bervorgehoben.

5) Schon Valisneri, ein Schüler Malpigh is (Istoria della generazione etc. Venedig 1721, deutsch von $\mathrm{C}$. Ph. Berg, Lemgo 1739) äusserte sich dahin, dass der Eintritt der Spermazellen ins Ei ausser aller Analogie mit den stetigen Entpuppungen der Insekten sei, er entspräche gerade einer rückschreitenden Motamorphose. 
geschieht überhaupt nicht alles in der Entwickelungsgeschichte direkt, indem mancher getane Schritt zurückgetan wird (Roux, S. 59); als Beispiele können ausser der Schilddrüse, Kiemenspalten und Kiemenarterien, Chorda dorsalis, Hypophysis, Zirbeldrüse angeführ werden.

m) Die malignen Geschwülste haben ihre volle Analogie in einer anderen Erkrankung, die zwar in eine ganz andere Kategorie eingereiht ist, aber, im Grunde genommen, ebenfalls eine zelluläre Neubildung vorstellt, nur dass sie sich in einer anderen Zellart etabliert, nämlich in den Blutzellen. Ich meine die progressive perniziöse Anämie, die eigentlich ein Carcinom der Blutzellen ist, indem eine massenhafte Zellvermehrung Hand in Hand mit einer Rückdifferenzierung der Zellen (kembaltige Erythrozyten als Jugendstadium der kemlosen usw.) einhergeht; und zwar nehmen hier die charakteristischen Erscheinungen, ähnlich wie beim Carcinom, progressiv zu. Auch das sogenannte Chorioepithelioma malignum ist ein Analogon für maligne Geschwülste ${ }^{1}$ ); das gewöhnliche Myxom der Plazenta ist - sagt Virchow ${ }^{2}$ ) - nicht bloss für die Schleimgeschwulst, sondern auch für die Geschwulst überhaupt von höchster Bedeutung.

Aber nicht die Anaplasie ist die Ursache der foudroyanten Zellvermehrung, sondern gerade die Zellvermehrung ist, wie. ich mich bemüht habe zu beweisen ${ }^{3}$ ), das Primäre, die Ursache von neotenischen Erscheinungen beim Carcinom. Dementsprechend muss die Entdifferenzierung mit der Zeit immer stärker werden, was wir in der Tat auch daraus sehen, dass die Metastasen, d. h. derjenige Zustand, wo die Entdifferenzierung und Desintegration den höclisten Grad erreichen, indem die Zellen sich dann überall adaptieren, nie von Anfang an oder in Bälde auftreten, sondern erst nach geraumer Zeit.

Das Yerdienst Hansemanns (übrigens hat bereits vor ihm dasselbe Ménétrier nachgewiesen) ist, hervorgehoben zu haben, dass die Funktion der Zelle bei ihrer Entdifferenzierung progressiv abnimmt. Doch kann die Entdifferenzierung beim Carcinom, wie ich nachgewiesen habe, ohne weiteres scbon auf deduktivem Wege, bloss aus der vermehrten Propagationsfähigkeit der Carcinomzellen eruiert werden. Ménétrier ${ }^{4}$ ) und nach ihm Hansemann und Hauser haben für dieses selbstverständliche Postulat auch tatsächliche Daten angeführt. So gingen also praktische Beobachtungen, wie oft, so auch hier, der theoretischen Grundlage voraus.

1) Besonders charakteristisch ist hier anch die Zugehörigkeit zu dem phylogenetisch niedrigen Schleimgewebe.

2) Geschwülste, S. 405 .

3) „Das Carcinom". 1898.

4) Des polyadénomes gastriques et de leurs rapports avec le cancer de l'estomac. Arcb. de phys. 4. S. I. 1888. 
v. Hansemann 1) sagt: „Es entsteht nur dann ein maligner Tumor, wenn der Wucherungsreiz eine anaplastische Zelle trifft". Weiter: "Wer die Aetiologie der Krebse nachweisen will, der muss zeigen, dass die ätiologischen Momente im Stande sind, nicht nur eine Wucherung, sondern auch eine Anaplasie zu erzengen." "Woher aber die anaplastische Zelle entsteht, wodurch sie entsteht, weshalb sie entsteht - diese Frage wirft v. Hansemann nicht auf, und dieser Frage liegt die Aetiologie zu Grunde - denn, sobald ich die Entstehung der veränderten Parenchymzellen der Krebsgeschwulst kenne, brauche ich keine Erklärung mehr für ihr weiteres Wachstum, da es wohl selbstverständlich ist, dass der Faktor, der die normalen Gewebszellen zu 'anaplastischen Zellen (Krebszellen) umgewandelt bat, auch ihr weiteres Wachstum und Vermehrung bedingen wird", sagt ganz richtig Feinberg' ${ }^{2}$. Mit anderen Worten, „beziehe sich die Anaplasie nach v. Hansemann, ausschliesslich auf die Morphologie und die Physiologie des Tumors, aber nicht auf die Aetiologie; die Anaplasie könne die Wachstumsenergie nicht erklären."

Ich glaube, dass meine Auseinandersetzungen diese Frage vollständig geklärt haben. Ich habe bewiesen (was Hansemann u. A. für unerklärt hielten), dass der Reiz hier „im Stande ist, nicht nur eine Wucherung, sondern auch eine Anaplasie zu erzeugen". Ich habe gezeigt, dass hier diejenigen Faktoren, die eine Wucherung erzeugen, auch die Anaplasie hervorrufen müssen; ich habe, laut der Forderung Hansemanns, erklärt, "woher, wodurch und weshalb" die anaplastische Zelle entsteht. Feinberg hatte Recht, wenn er meinte, dass in der Kenntnis der Entstehung der Veränderung in den Krebszellen (im Vergleich mit normalen Zellen, aus denen sie hervorgegangen) die Erk]ärung für ihr weiteres Wachstum gegeben ist. Hausemann hat dagegen Unrecht, wemn er gegen Ribbert behauptet, dass ein maligner Tumor durch einen Wucherungsreiz nicht auf das normale Gewebe, sondern nur auf eine anaplastische Zelle entstehe: denn derselbe Reiz, der die anaplastische Zelle zur Krebszelle macht, macht auch aus der normalen eine anaplastische Zelle. Ich habe gezeigt, wie sich der. Leser wohl erinnern wird, dass durch Vergrösserung der Nahrungszufuhr die Zellen sich immer mehr und mehr teilen und stärker vermehren müssen und dass diese vermehrte Teilung von neotenischen Eigenschaften begleitet wird und werden muss. Die grössere Nahrungszufuhr wird, wie ich schon früher ${ }^{3}$ ) gezeigt habe, durch Reizung der Zellen verursacht. Es war schon längst aus der Praxis bekannt, dass Krebsgeschwülste auf Grund chronischer unterbrochener Reize entstehen.

1) Geschwülste.

2) Verhandlungen des Comités für Krebsforschung. Heft I. S. 43.

3) Das Carcinom. S. 5-7, 13. 
Ich habe auf theoretischem Wege bewiesen 1 ), dass die Entstehung einer starken Zellvermehrung und die Annahme neotenischer Eigenschaften denkbar ist nur bei chronischen und zugleich intermittierenden Reizen, und somit eine theoretische Grundlage für die bis jest nur aus der Praxis bekannte Tatsache geliefert. Durch chronisch-intermittierende Reize entsteht stärkere Nahrungszufuhr, durch grössere Nahrungszufuhr - stärkeres Wachstum mit gleichzeitigem Auftreten kleinerer und phylogenetisch minderwertiger Zellen, d. h. Entdifferenzierung. In allen derartigen Prozessen ist vor allem das Wachstumstempo geändert, es wird beschleunigt. Dass hier allgemeinere Gesetze walten, geht daraus hervor, dass alle bei dem im Kapitel I aufgestellten Gesetz erwähnten Momente beim Carcinom regelrecht wiedererscheinen.

1. Die Krebszellen nehmen an Zahl immer mehr und mehr zu.

2. Das Wachstumstempo wird immer schneller; die Zeitdauer bis zum Auftreten der Teilung wird immer geringer.

3. Die Zellen nehmen allmählich an Grösse ab.

4. Sie leben immer kürzer und kürzer.

5. Sie werden funktionell und anatomisch minderwertiger ${ }^{2}$ ).

Nun entsteht die Frage: warum entwickeln sich aus diesen entdifferenzierten Zellen keine höhere Zellen, wie es z. B. beim Embryo der Fall ist oder warum entstehen beim Embryo aus den wenig differenzierten Zellen höhere Zellen, beim Carcinom aber nicht?

Wir haben geseben, dass die Entstehung böherer Zellen an Nahrungsverminderung gebunden ist. Beim Carcinom wird aus angegebenen Gründen ${ }^{3}$ ) die Nahrungszufuhr immer grösser, beim Embryo tritt im Gegenteil nach einer gewissen Zeit Verminderung der Nahrungszufuhr (welche sich auch in Verminderung des Wassergehaltes äussert) ein. Es ist theoretisch nicht ausgeschlossen, sagt Mühlmann, dass eine Muskel-, Nervenoder Bindegewebszelle mit der Verbesserung der Eruährungsverhältnisse sich wieder in eine Blastzelle (Ur-, Keim-, Plasma-, Wanderzelle) umwandeln kann. Würde beim Embryo die Ernährung ständig unverhältnismässig stark zunehmen, so würde er sich nicht differenzieren können, im Gegenteil würde daraus ein Komplex von allereinfachsten Zellen entstehen. Dagegen kommen die Zellen im Carcinom kaum jemals zur Ruhe in Bezug auf Proliferation, sie teilen sich bald nach ihrer Entstehung wieder und finden so keine Zeit ihre spezifischen Eigenschaften auszubilden (vergl. auch Lubarsch, S. 302).

1) Ibid. S. 6-9.

2) Nach Hauser (S. 299) ist das Dunklerwerden des Protoplasmas und der stärkere Chromatingehalt der Kerne in den Krebszellen auch ein Zeichen der Anaplasie.

3) S. Das Carcinom. 1898. S. 5-7. 
Ad 4. Warum entsteht das Carcinom gewöhnlich im höheren Alter? Dafür gibt es mehrere Ursachen:

a) Mit zunehmendem Alter nimmt die Neigung zu Geschwulstbildungen erheblich zu. Auch im normalen Körper gibt es eine gewisse Periodizität in Bezug auf das Wachstum (z. B. Haare im Winter, Zähne, Knochen, Mamma). So dauert auch die Zellenproduktion bei gewissen Organen während der ganzen Fötalperiode, wie bei den meisten Drüsen; bei anderen zieht sich dieselbe sogar durch die ganze Wachstumsperiode hindurch, wie bei den Knochen, Knorpeln, Zähnen; bei noch anderen endlich zeigt sich dieselbe selbst im ausgewachsenen Organismus, wie bei den weissen und roten Blutzellen und den Zellen der absondernden Drüsen (Hoden-, Milchund Talgdrüsen). In noch ausgedehnterem Grade finden wir diese Erscheinung in der Pflanzenwelt: das Wachstum der Pflanzen ist ja an Perioden gebunden - die perennierenden Gewächse erzeugen alle Jahre Blätter, Blüten und Früchte. - Für die Periodizität spricht auch das Wachstum der Dermoide und Teratome.

b) Es existiert ein gewisser Antagonismus zwischen dem Wachstum einzelner Körperteile. Diese Erscheinung tritt bei den Geschwülsten besonders prägnant auf: das Lipom eines noch so abgemagerten Phthisikers ist in seinem Fettgehalt unverändert, auch bei Inanition tritt kein Rückgang des Carcinoms ein. Ist einmal ein Ueberwiegen von Wachstum in einem Körpertheil eingetreten, so strömen die Körpersäfte überwiegend nach dieser Stelle hin. Auf den nutritiven Antagonismus hat auch Virchow ${ }^{1}$ ) aufmerksam gemacht, der z. B. in der Polysarzie der Azephalen

1) Zellular-Pathologie. Dieser Antagonismus ist auch deutlich zu erkennen in Bezug auf Geschlechtsorgane. Je höher wir im Tierreich emporsteigen, desto mehr treten die Geschlechtsorgane dem Volumen nach gegen das Gewicht des Körpers zurück: während sie beim Weib $0,29 \mathrm{pCt}$. des Körpergewichtes ausmachen, giebt es Tiere, deren Keimzellen $1 / 4$ oder $1 / 2$ des Körpers bilden oder sogar den übrigen Körper an Volumen übertreffen. So nehmen auch beim Rheinlachs während des 6-9 Monate dauernden Fastens beim Aufenthalt im Rbein die Muskeln enorm an Volumen ab, während die Geschlechtsorgane eine ausserordentliche Entwickelung erlangen. Hier findet also ein Kampf um die Nahrung zwischen den Gewebselementen der Geschlechtsorgane und der Muskulatur statt. Der Eierstock wächst dabei von $1 \mathrm{pCt}$. auf $23 \mathrm{pCt}$. des Körpergewichtes heran, die Rumpfmuskulatur verliert dabei $43 \mathrm{pCt}$. ihres Gewichtes und der zurückgebliebene Rest ist um 23 pCt. an Eiweiss verarmt. Auch Magen, Darm, Leber etc. verlieren dabei bedeutend an Gewicht (Miescher-Rüsch). Es wachsen also die Geschlechtsorgane auf Kosten der von den anderen Organen gelieferten Stoffwechselprodukte. Auch zeichnen sich unfruchtbare Frauen gewöhnlich durch grösseren Fettansatz aus. So kommt (Roux, S. 106) die Osteomalacie bei Frauen, die jahrelang stillen, vor, indem bei der fortwährenden Abfuhr von Ca-Salzen durch die Milchdrüsen den Knochen die Ca-Salze vorweggenommen werden aus der Nahrung. Es 
und der Zwergkretins zum Vorschein kommt. So ist auch Hypertrichosis gewöhnlich mit einer mangelhaften Entwickelung des Gebisses verbunden; Geweih- und Zahnbildung scheinen sich gegenseitig auszuschliessen; bei Entfernung der Augen wird der Olfaktorius mächtiger. Dasselbe gilt auch für das psychische Leben: auf der Ausbildung einzelner Sinne auf Kosten anderer beruht das Blindmachen der Musiker in Altertum.

Was hat diese ganze Episode über den Antagonismus zwischen der Ernährung einzelner Körperteile für unsere Frage für Bedeutung?

Beim jugendlichen, wachsenden Organismus verlangen beinahe alle Zellen eine stärkere Nahrungszufuhr, deren Anziehung an einer Stelle nicht bedeutend grösser als an einer anderen sein kann; die Zellen befinden sich überall fast in gleichem Nahrungsbedürfnis. (Das Wachstumstempo der Zellen des kindlichen Organismus ist nicht so verschieden von denen eines Carcinoms.) Es kann also eine Vermehrung des Nahrungsüberflusses an einer Stelle nicht zu einer hochgradigen Proliferation der entsprechenden Zellen führen, weil von allen Seiten her die Nabrung in Anspruch genommen wird. Bei alten Leuten aber, wo die Ernährung der einzelnen Teile im Vergleich mit kindiichem Alter eine geringe geworden ist, kommt es, wenn eine Perturbation an einer Stelle eintritt, sehr leicht zum Sieg im Kampfe um die Ernährung seitens derjenigen Gewebe, die verhältnismässig reichlichere Ernährung erbalten - umsomehr als ein gewisser Ueberfluss von Nährmaterial infolge von Verminderung des Nahrungszutiusses zu den atrophischen Geschlechtsorganen sich etabliert. Es wird also im höheren Alter schon eine geringe Störung leicht zu einem grösseren Nahrungsüberfluss an der gereizten Stelle und zwar auf Kosten der übrigen Körperteile führen müssen.

c) Die Theorie von Thiersch, dass die Ursache der im höheren Alter (beim Carcinom) eintretenden hochgradigen Proliferation des Epithels in findet hier also ein Kampf der Milchdrüsen mit den Knochen dadurch statt, dass die Zellen der ersteren die Ca-Salze stärker aus dem Transsudat anziehen als die Knochengrundsuobstanz. - Schon Canstatt (Die Krankheiten des höheren Alters und jhre Heilung, Erlangen 1839) sah die Geschwülste als Produkte des durch den Schwund der Generationsorgane entarteten Generationstriebes an - eine Ansicht, die von vielen neueren Autoren vertreten wird. - Nach Morpurgos Untersuchungen sind während des Inanitionszustandes gerade in den Geschlechtsorganen besonders lebhafte Kernteilungsprozesse zu beobachten (Kassowitz. Biologie. Bd. II. S. 71).

Dieser Antagonismus ist auch deutlich in der Pflanzenwelt erkennbar: während das einjährige Kraut Blüten trägt und Samen zur Reife bringt, ist der junge Baum immer noch beschäftigt, seine ursprünglich saftige Axe in eine dichte faserige Substanz umzuwandeln; und Jahr für Jahr verbraucht derselbe, indem er beständig das Gleiche tut, bei dieser Gelegenheit eine Menge von Näbrstoffen, welche die aufeinanderfolgenden Generationen des einjährigen. Gewächses zur Bildung ron Früchten verwenden konnten. 
der Atrophie des Bindegewebes zu suchen ist, ist nicht richtig 1). Nicht auf der Wachstumsenergie des Epithels beruht die Ursache der Carcinombildung, sondern gerade auf der mangelnden Regenerationsfähigkeit des Epithels bei alten Leuten. Dass das Epithel im höheren Alter an Wachstumsintensität abnimmt, das beweisen uns u. a. auch die Ulcera varicosa der alten Leute. Würde das Epitbel dieselbe Regenerationskraft bei alten Individuen besitzen, wie es bei jungen der Fall ist, so würde es zu keinem chronischen Reiz und folglich auch nicht zum Carcinom kommen können. Würde wirklịch die Wachstumsenergie des Epithels die Carcinombildung verursachen, so müsste im Gegenteil der Krebs bäufiger sein im kindlichen Alter. Gerade dadurch, dass die Epithelien bei älteren Leuten sich schwieriger regenerieren, wird der Reiz unterhalten, er wird immer und immer grösser, indem der einmal gesetzte Defekt nicht schnell heilt und dadurch Gelegenheit zu weiterer Reizung gegeben wird. Der einmalige Reiz wird auf diese Weise zum chronischen. Diese Reizung führt zu grösserer Nahrungszufuhr an den gereizten Stellen, die auf Kosten der übrigen Körperteile erfolgt und mit der Zeit immer mehr und mehr Ueberband nimmt und so zu einer enormen Zellwucherung, wie es das Carcinom darstellt, führt. Im höheren Alter bedeutet schon ein geringer lokaler Unterschied in den Ernährungsverhältnissen viel: das Widerstandsvermögen jeder Krankheit gegenüber ist um so grösser, je niedriger der Grad der phyletischen Entwicklung des Organismus ist - was sowoll auf Zellen wie auch auf Gewebe und ganze Individuen sich bezieht. Es erlangt also infolge von Reizung das Epithel, welches im höheren Alter sich durch eine geringere Regeneration auszeichnet, die Eigenschaft, sich stark zu vermehren. Auch in jüngerem Alter würde das Epithel seine Regenerationskraft bis zur Carcinombildung steigern können, aber der Defekt wird im Gegensatz zum höheren Alter schnell gedeckt, ehe noch die Zelien durch Reizung (d. h. hier schnelle Teilung) entdifferenziert werden. Die Eigenschaft einer stärkeren Proliferation erlangen die Epithelien im böheren Alter, wie wir gesehen haben, eben dank der Abuahme der ursprünglichen Regenerationskraft. Lnsere Erklärung findet darin ihre Stütze, dass wenn einmal das Carcinom sich doch bei jugendlichen Individuen entwickelt, es dann unvergleichlich schneller wächst und ausgedehntere Metastasen als bei ganz alten Menschen macht ${ }^{2}$ ): denn wenn einmal der Reiz so intensiv gewesen war, dass die Regeneration des Epithels nicht hinreichte, den Defekt binnen

1) Uebrigens war Canstatt (l. c.) der erste, welcher das involutive Verbalten des Bindegewebes zur Erklärung der Wachstumsenergie der Geschwülste (Pseudoplasmen) benutzt hat.

2) Dasselbe Verhalten zeigen auch die Mäuse bei künstlicher Transplantation von Carcinom. Bashford, Lancet. 1905. Decemb. 9. p. 1671. 
kurzer Zeit zu decken, so wird dadurch der Reiz weiter unterhalten und infolge der grossen Regenerationskraft des kindlichen Organismus die Vermehrung đer Zellen eine sehr grosse sein müssen. So erklärt auch Maly ${ }^{1}$ ) die Entstehung des Carcinoms bei nicht heilender Cervixerosion, indem das neugebildete Gewebe wieder vernichtet wird (cf. S. 606).

Würde das Epithel im höheren Alter die Regenerationskraft nicht teilweise, sondern gänzlich verloren haben, so würde auch kein Carcinom entsteben, denn dann würden einfach die Epithelien zu Grunde gehen und durch Bindegewebe (Narben) ersetzt werden. Würde das Epithel im höheren Alter dieselbe Regenerationskraft besitzen wie in der Jugend, dann würde auch der Defekt schnell gedeckt werden und dadurch keine Möglichkeit für die Entstehung eines chronischen Reizes gegeben sein, ohne welchen sich kein Carcinom entwickeln kann. Aber in dem Fall, wo das Epithel die Regenerationsfähigkeit nur in einem gewissen (rrade (nicht aber gänzlich) verloren hat, wie es im höheren Alter der Fall ist, entsteht eben eine starke Wucherung, weil auf diese Weise der Reiz nicht verschwindet; einmal aufgetreten, wird er von selbst unterhalten. So sehen wir auch, dass, wie stark auch der Reiz sein mag bei Ganglienzellen oder bei den Zellen der quergestreiften Muskulatur ${ }^{2}$ ) (welche bekanntlich sich nicht regenerieren), dieselben nie so weit zurückdifferenziert werden können, dass sie eine Vermehrungsfähigkeit erlangen: man hat bis jetzt noch nie eine zelluläre Variation eines Rhabdomyoms oder eines echten Neuroms gesehen. Würde also das Epithel absolut nicht regenerationsfähig sein, so würde keine zelluläre Variation des Tumors entstehen können; die Anlage zur Carcinombildung liegt in der geringen Regenerationsfähigkeit des Epithels. Im höheren Alter nimmt nicht das Epithel, sondern gerade das Bindegewebe an Mächtigkeit zu. So sehen wir im höheren Alter eine Hypertrophie der Nase und der Ohrmuschel eintreten eben infolge von Bindegewebswucherung. Aus dem Obengesagten ist es klar, worin das Falsche in der Theorie von Thiersch lag.

Ist aber das Wachstum des Carcinoms von Regenerationsfähigkeit der betreffenden Zellen abhängig, so fragt sich nun: Warum entsteht so selten ein Carcinom primär in der Leber? Wir haben gesehen, dass zur Entstehung der Wucherung eine Schädigung nötig ist, die also da am häufigsten vorkommen wird, wo der Körper äusseren Einflüssen ausgesetzt ist. Das ist der Fall eben in allen unseren Hautund Schleimhautepithelzellen, d. h. bei den Zellarten, die in direktem Kontakt mit der Aussenwelt sich befinden. Die Leber liegt aber so geschützt im Körper, dass sie selten einer Gelegenheit, geschädigt zu werden, ausgesetzt sein kann. Lebrigens hört, nach $M$ ühlmann ${ }^{3}$, die Leber schon relativ frühzeitig zu waobsen auf. In

1) Centralbl. f. Gynäkol. 1903. No. 27.

2) Es gibt also eine biologische Prädilektion und Immunität in bezug auf die Entstehung von Carcinom.

3) S. 132. 
der Leber eines erwachsenen Menschen sind, wie man weiss, bereits sehr frühzeitig keine Kernteilungsfiguren mehr vorhanden. „Das absolute Wachstum ist frühzeitig schon so gering, dass es nur selten zu Zellvermehrung kommt. Es lässt sich auch nachweisen, dass in der postembryonalen Zeit die Leberzelle auch an Grösse kaum zunimmt." Ueberdies ist die Frage über die Regeneration der Leber noch unentschieden ${ }^{1}$ ).

Ad 5. Aus der Tatsache, dass nicht alle, deren Zellen einem chronischintermittierenden Reiz ausgesetzt sind, an Carcinom erkranken, den Schluss zu ziehen, dass wir die eigentliche Ursache der Carcinomentstehung nicht kennen, halte ich für ganz unrichtig. Denn dann müssten wir auch sagen, dass wir überhaupt über die Aetiologie aller infektiösen Krankheiten nichts wissen, denn z. B. bei Choleraepidemien erkranken doch nicht alle an Cholera. Also von diesem Gesichtspunkte ausgehend ist unsere Keuntnis der Carcinomentstehung auf jeden Fall nicht geringer als z. B. die des Typhus und der Cholera.

Ad 6. Das Wachstum des Carcinoms in die Tiefe ist auch keine spezifische Eigenschaft des Carcinoms. Das ist derselbe Prozess wie bei der Embryonalentwicklung, ein Prozess, der sowohl im Lupus, in den von Hauser beschriebenen atypischen Epithelwucherungen in der Narbe von Mlagengeschwüren, als auch in den bereits vielfach beschriebenen unschuldigen Drüsenwucherungen bei einfachen atrophischen Prozessen, z. B. an der Zunge, im Uterus, in der Mamma sein Analogon hat ${ }^{2}$. In gewissen Perioden kommt ein solches Wachstum normaliter zum Vorschein, so z. B. bei Neubildung ganzer Drüsenläppchen in der Laktation, beim Ersatz drüsiger Bestandteile der Uterusschleimhaut nach der Menstruation usw.

Während der Embryonalentwicklung begegnen wir auf Schritt und Tritt ähnlichen Bildungen: die Drüsenbildungen im Verlaufe des Darmkanals, die Bildung von Schlundspalten, die Anlage der Kiemenbögen und der Lungen, der

1) Vergl. die Einwendungen seitens Cornils und Carnots auf Grund ihrer Versuche (Bulletin de l'Acad. de médecine. 1897-98; Presse méd. 1898; Arch. de méd. expér. 1898; Semaine méd. 1898; auch Carnots La régénération d'organes. Paris 1899 . pp. 16, 74-84, 93). Carnot unterscheidet eine Restitution der Form und Restitution der Funktion. Die erste ist um so vollständiger, je niedriger das Tier; die Restitution der Funktion aber persistiert auch bei höheren Tieren, wo sie die Hauptform darstellt. So ist die Regeneration der Leber (wie des Pankreas, der Niere usw.) eine funktionelle und keine morphologische, die ursprüngliche Form wird nicht restituiert; die Regeneration tritt nicht lokal auf. In bezug auf Lunge vergl. meinen Aufsatz im Archiv f. experim. Pathol. u. Pharmakol. Bd. LV. 1906: "Die Folgen von Lungenexstirpation".

2) Der Unterschied im Gegensatz zu Carcinom besteht in den hier stets fehlenden Reaktionserscheinungen (Edinburgh med. Journ. 1898. - Tietze, Deutsche Zeitschr. f. Chirurgie. 1904. Bd. 75. - Zieler, Med. Gesellsch. in Göttingen, 2. V. 1901. Ref. Deutsche med. Wochenschr. 1901. No.22. - Schaffer, Wiener Sitz.-Berichte. Abt. III. Bd. CVI). 
Thymus, der Schilddrüse, die Bildung der Nluskelsegmente usw. - alles das ist nichts anderes als Faltenbildung. Die Gastrula stellt eine Faltenbildung erster Ordnung, die Medullaranlage und die Mesodermbildung Faltenbildung zweiter Ordnung dar. Auch die Bildung der Eihüllen gehört hierher als Faltenbildung erster Ordnung, ebenso die Anlage der Zähne ${ }^{1}$ ).

$\mathrm{Da}$, wie wir gesehen haben, die Carcinomzellen entdifferenzierte Zellen sind, so kann es uns nicht wundern, dass sie auch die Eigenschaft der embryonalen Gebilde in bezug auf die Wachstumsrichtung erlangen. Also besitzt das Carcinomgewebe auch hier neotenische Eigenschaften, d. b. Aehnlichkeit mit phylogenetisch niedriger stehenden Prozessen - da diese Epitheleinsenkungen, Falten, das Wachstum nach innen zu, zu welchem sich die Abschnürung hinzugesellt, normal auf einer niederen Entwicklungsstufe der Tiere gebildet werden ${ }^{2}$ ).

Die Ursachen der Faltenbildung können beim Carcinom keine anderen sein als bei normalen Geweben. Die Falten entstehen, nach Driesch, bei starker Vermehrung der Zellen durch Widerstände infolge von Raumverengung ${ }^{3}$ ).

Wie die Aenderung der Wachstumsrichtung zustande kommt, habe ich bereits auseinandergesetzt ${ }^{4}$ ). Die Bedeutung ist stets dieselbe: das Erlangen einer grösseren Oberfläche. Dieses Ziel wird beim Carcinom mit der höchsten Konsequenz erreicht, indem in den gefalteten Gebilden wieder Faltungen zweiter Ordnung, daraus Faltungen dritter Ordnung usw. entstehen. Dieses Prinzip - das Erlangen einer grösseren Oberfläche findet seinen Ausdruck auch in der Mehrschichtigkeit des Epithels der Drüsen. Durch diese Mebrschichtigkeit bekommen die drüsenartigen Carcinombildungen eine Aebnlichkeit mit der oberfiächlichen Epidermis- oder Mukosaschicht, aus der sie hervorgegangen sind.

Wenn man von einer Aenderung der Wachstumsrichtung beim Carcinom spricht, so darf man nicht vergessen, dass dabei die Teilungsrichtung der Zellen nicht geändert wird. In welcher Ebene die Richtung der Epithelzellen auch verläuft, stets erfolgt die Teilung in der Richtung nach der freien Oberfläche, d. h. gegen die Aussenfläche zu (einerlei, ob es sich um Epidermis oder Vukosa handelt); nur inbezug auf die relative Lage zu den übrigen Geweben

1) Nïhlmann, S. 55 .

2) Die Abschnürung ist bekanntlich ein wesentlicher Faktor der Entwicklong. Die durch die Faltenbildung in der Mediallinie des äusseren Keimblattes entstehende Medullarlinie wird zu einer Röhre und löst sich vom Muttergewebe ab, ebenso wie die in der Mediallinie des inneren Keimblattes entstehende Chorda sich ablöst. Hand in Hand mit der Vermehrung der Zellen und den Faltenbildungen gehen Abtrennungen der gebildeten Falten.

3) Die Hohlräume bilden sich infolge mangelhafter Ernährung centraler Teile gegenüber den peripheren (z. B. Gastrula, Müblmann S. 53).

4) S. „Das Carcinom". S. $24 \mathrm{ff}$. 
des Körpers ist dies als eine Richtungsänderung zu bezeichnen. Es ist auch übrigens, strikte ausgedrückt, kein Wachstum nach unten, sondern nur Faltenbildung, und zwar durch Einsenkung, nicht durch Hervorstülpung, und dadurch kommt erst sekundär das Wachstum nach unten zustande. Es entsteht noch die Frage, warum die Faltenbildung in der Richtung nach unten und nicht nach oben erfolgt, d. h. warum eine drüsenähnliche und nicht eine papillenähnliche Form entsteht?

Auch das ist leicht zu erklären. Papillenbildungen sind nur möglich da, wo das Bindegewebe besonders gut ernährt wird; jedenfalls bildet die Einsenkung in die Tiefe bessere und sichere Bedingungen für gute Ernährung als eine Entfernung von der Eruährungsquelle. Bei Drüsenbildung kommt das Epithel direkt in die Nähe dieser Ernährungsquelle zu liegen, bei Papillenbildung müsste es diese Ernährungsquelle mit sich mitziehen ${ }^{1}$ ). Offenbar ist für die Enstehung von Zellwucherung der erstere Modus günstiger als der zweitè.

Ad 7. Aus welcher Schicht geht das Carcinomgewebe hervor?

Auf Grund der Topographie der verschiedenen Epithellagen in den Kankroidperlen habe ich bewiesen ${ }^{2}$ ), dass die Carcinomwucherung von der Epithellage ihren Anfang nimmt und dass die weitere Entdifferenzierung dann eintritt, wenu die wuchernden Zellen an die untere Grenze, an die Basalmembran gelangen, d. h. an diejenige Schicht, die ebensogut als Anfangsstation des Bindegewebes wie des Epithels betrachtet werden kann ${ }^{3}$ ).

1) Diese Aenderung der Wachstumsrichtung ist leicht hervorzurufen, so z. B. durch Belichtung (Herbst, Winkler u. a.). Auch durch Einwirkung gewisser chemischer Körper, z. B. des Lithiums, erzielte Herbst die Bildung von „Exogastrula", d. h. der Darm wuchs dabei nach aussen. Für dio normale Gastrulation ist eine ganz typisch geregelte Eigenschaft der Zellen hinsichtlich ihrer osmotischen Qualität die Bedingung. Vergl. auch Helene King, Arch. f. Entwickelungsmechanik. Bd. XVI. Heft 2. 1903.

2) Das Carcinom. S. 27, 28.

3) Das bezieht sich ebenso auf die äussere Haut, wie auf die Schleimhaut, was rom embryologischen Standpunkt leicht verständlich ist. In der Epidermis sind es die untersten Schichten, in denen allein Mitosen vorkommen, das sind diejenigeñ, die dem Bindegewebe am nächsten liegen. In mehrschichtigen Schleimhäuten liegen die Teilungsfiguren wie bei der Epidermis in den untersten Zellschichten. "Mitosen beim Dickdarmcarcinom findet man mehr im Fundus der Lieberkühn'schen Krypten als nach der Oberfäche zu und es scheint, dass die an der Oberfläche des Darmes verbrauchten Zellen hier unten ersetzt werden und allmählich hinaufrücken" (v.Hansemann, S. 31).

Die Umwandlung ron Zylinder- in Plattenepithel (z. B. bei Kankroiden der Gallenblase, der Bronchien, der Trachea usw.) und umgekehrt des Plattenepithels in Zylinderepithel, ist durch den Bau der geschichteten Epithelarten leicht zu erklären, indem die unteren Lagen des geschichteten Plattenepithels mehr zylin- 
Also nicht vom Bindegewebe, wie Ribbert irrtümlich meint, nimmt das Carcinom seinen Anfang, sondern vom Epithel. Das Entstehen durch primäre Wirkung des Bindegewebes würde auch kaum ein Analogon in anderen pathologischen Prozessen haben, während das Entstehen primär aus dem Epithel, entsprechend dem auch in vielen anderen Beziehungen sich äussernden Charakter der Carcinomzellen, sich mit den embryonalen Verhältnissen in Uebereinstimmung befindet. Denn auch während der Embryonalentwickelung ist es das Epithel, welches zuerst wuchernde Zapfen in das Mesoderm treibt; erst nach einiger Zeit wird auch das Mesoderm aktiv und treibt seinerseits Wucherungen in das Ektoderm. Das Ovarium ist auch ein gutes Beispiel in diesem Sinne.

Dass die Wucherung vom Epithel und nicht vom Bindegewebe ausgeht, beweist, wie gesagt, unstreitig die Konfiguration der Perlenbildung ${ }^{1}$ ). Aus den sonst unansehnlichen Gebilden lassen sich also wichtige, kardinale Schlüsse ziehen. Das Entstehen aus dem Epithel beweisen auch, wie Orth richtig bemerkt ${ }^{2}$ ), die sog. Lymphgefässkrebse, z. B. der Lunge, des Uterus: denn da in den Lymphgefässen das Stroma überhaupt fehlt, so bestehen hier die Carcinome, die also auch keinen alveolären Bau haben, lediglich aus heterotop wuchernden Epithelien. Ebenso finden wir in Geschwulstthromben frisch entstandene Mitosen, das Bindegewebe fehlt vollkommen, indem der Thrombus aus lose zusammenhängenden Zellen besteht.

Ad 8. Wie lange mindestens der Reiz dauern muss, damit ein Carcinom entstehe, sieht man aus der Wirkung der Röntgenstrablen und des Radiums. Bekanntlich wird dadurch oft die Carcinomwucherung zum Schwinden gebracht. Aber umgekehrt hat man über mehrere Fälle von Carcinomentwickelung nach Röntgen- resp. Radiumbehandlung berichtet ${ }^{3}$ ). Die Zeit also, die nötig ist, damit ein Carcinom entstehen könne, ist ziemlich kurz - sie kann nur wenige Monate betragen. Dieses Auftreten von Carcinom nach Einwirkung von Röntgenstrahlen zeigt uns zugleich in unzweifelhafter Weise, dass die Ursache der Carcinomentwickelung ein chronischer Reiz bildet. Das ist der beste experimentelle Beweis dafür.

drische, die des zylindrischen mehr Plattenform haben. Wie bei einem Geschwür die indifferente Schicht, von der aus früher das Wachstum nach unten erfolgte, zur äusseren wird und dann, die Rolle der Epidermis übernehmend, nach aussen zu wachsen beginnt, so nimmt beim Carcinom das oberfächliche Epithel die Eigenschaften des unten liegenden jüngeren Epithels von embryonalem Charakter an.

1) Cf. Anmerk. 2. S. 627.

2) Heilungsvorgänge an Epithelien. Zeitschr. f. Krebsforschung. I. 1903.

3) Beck, Münchner med. Wochenschr. 1905. No. 7. - Lancet. 1904. II. p. 1172 und 1902. p. 1454. - M. O. Wys s, Beitr. zur klin. Chirurgie. 1906. Bd. XXIX. - Lassar, Berlin. Med. Ges. Sitzung rom 2. Juli 1906. 


\section{Dritter Teil.}

\section{Therapie.}

Lassen sich aus den biologischen Gesetzen irgendwelche Hinweise inbezug auf die Therapie folgern?

1. Vor allem müssen wir die Tatsache konstatieren, dass das Carcinom heilbar ist auch ohne unser Zutun; ebenso verschwinden Chorioepitheliome von selbst ${ }^{1}$ ). Fälle von geheilten Carcinomen nach Gastroenterostomie, auch nach Radiotherapie ${ }^{2}$ ) und bei Erysipel sind mehrfach publiziert und konstatiert worden; Hautcarcinome verschwinden nach Anwendung von As usw.

Weiter wissen wir, dass die Carcinome einen verschiedenen Grad von Bösartigkeit haben können, dementsprechend auch die Dauer ihres Bestandes eine verschiedene ist. Es gibt langsam wachsende und schnell wachsende Carcinome ${ }^{3}$ ). Wir haben auf der einen Seite Medullarcarcinome, auf der anderen die Skirrhen. Melanotische zelluläre Tumoren zeichnen sich ganz besonders durch ihre Bösartigkeit aus. Manche Carcinome bleiben 10 und 20 Jahre lang bestehen: erinnern wir uns an das Ulcus rodens. Auch die Harnblasenkrebse wachsen verbältnismässig langsam. Ebenso weist auch das Sarkom verschiedene Grade von Bösartigkeit auf: als Epulis barmlos, verläuft es als Knochensarkom der Extremitäten als bösartigste Krankheit ${ }^{4}$ ); hier grosszellige Spindelsarkome, da Rundzellen-

1) v. Velits, Zeitschr. f. Geburtsh. u. Gynäk. Bd. LVI.

2) v. Hansemann. - D'Arcy Power, Lancet. 1899. No. 4. p. 583; No. 11. p. 693. - Zangger, Korrespondenzbl. f. Schweizer Aerzte. Okt. 1904. - Lancet. 1905. 25. IX. p. 821. - Lancet. No. 822. 22. März. A. Marmaduke-Sheild, 2 Fälle. - A. Pearce Gould, Lancet 1902. März. p. 821.

3) Billroth hat Carcinomfälle von 18 jähriger Dauer beschrieben, William s berichtet über einen Fall von Ulcus rodens, in dem sich die Erkrankung vom 14. bis 36. Jahre, also über 22 Jahre lang hinzog.

4) Auch manche Sarkome der Parotis gehören zu den gutartigen. „Die alte Regel, dass an den langen Röhrenknochen die zentralen Sarkøme durchschnittlich viel gutartiger verlaufen, als die periostalen, lässt sich wohl für jede Sarkomart einzeln nachweisen, ganz besonders aber besteht dieses Verhältnis zu Recht, wenn man seinen Blick auf eine kleine Gruppe lenkt, die in ihrer reinen Form klinisch und histologisch eine Sonderstellung beanspruchen darf; die zentralen Riesenzellensarkome der langen Röhrenknochen oder schaligen myeologenen Sarkome oder Myeloide, wie sie Virchow genannt hat. Das sind in der Tat klinisch und histologisch gutartige Geschwülste, und in der Literatur findet man eine Anzahl Beispiele, wo sie mit bestem Erfolg mit möglichst konservativen Operationsmethoden behandelt wurden. Derartige bösartige, in der Literatur verzeichnete Fälle sind 
sarkome. Endotbeliome gehören zu den weniger gefährlichen zellulären Tumoren.

Die Bösartigkeit als Ausdruck der gesteigerten Wachstumsenergie äussert sich auch in der Entstehung von Hetastasen, für welche die Bedingungen mit der Zeit immer günstiger werden müssen, wie das aus unseren früheren Betrachtungen hervorgeht, d. b. die Neigung zur Metastasenbildung läuft der Stärke der Zellvermehrung parallel. (Das Carcinom, S. 23.) Auch v. Hansemann kommt, obwohl auf anderem Wege, zu ähnlichem Schluss, nämlich dass die Neigung zu Metastasenbildung mit dem Grad der Anaplasie zunimmt und dass ein Parallelismus zwischen dem Grad der Anaplasie und dem Grad der Bösartigkeit besteht'1).

2. Wir haben gezeigt, dass die Wucherung der Carcinomzellen mit der Entdifferenzierung derselben eng verknüpft ist, und unsere nächste Aufgabe wäre zu untersuchen, ob es möglich ist, dass diese entdifferenzierten Zellen sich wieder differenzieren. Wir haben bereits geseben, dass die Differenzierung durch Verminderung der Nahrungsaufnahme erreicht wird (cfr. S. 600). r. Seeland hat in seinen von uns erwähnten Versuchen nachgewiesen, dass die Jugendzeit der Tauben und Hühner, welche er zeitweilig fasten liess, eine längere war als normal; sie erreichten die Zeit der Geschlechtsreife erst später und ebenso fing deren Körpergewicht, nachdem es einmal den Höhepunkt erreicht hatte, erst später an, abzunehmen. Kurz es liege, sagt Jickeli2), in der Einfalt jenes Bäuerleins, welches seinem Esel das Essen ganz abgewöhnen wollte und dem der Erfolg des Experimentes nur durch den Tod des Esels verdorben wurde, immerhin ein Fünkchen Wahrheit.

Also durch Verminderung der Nahrungsaufnahme kann man die Lebensdauer verlängern. Diese Nahrungsverminderung ermöglicht auch die Differenzierung der Gewebe: wo die lebende Masse sich teilt und wieder teilt, dort kann keine beträchtlicbe Arbeitsteilung sich ausbilden (Spencer). Wir haben gesehen, wie das Muskelgewebe und die Interzellularsubstanz dort entstehen, wo die Nahrung geringer wurde. Das ist auch die Ursache der Entstehung von Bindegewebe aus Epithelzellen im Laufe der Embryonalentwickelung. Alberts sagt in seiner Carcinommonographie, dass solange wir nicht wissen werden, warum im Laufe der normalen Entwickelung aus dem Epithel das Bindegewebe entsteht, so lange werden wir auch die Ursache der Carcinombildung nicht kennen, denn beim Carcinom gehen,

keine reine Riesenzellensarkome, sondern Mischformen" (Schmieden, Ueber den Wert der Theorie von der traumatischen Geschwulstgenese. Dtsch. med. Wochenschrift. 1902. No. 1.

1) Es gibt aber infiltrierend wachsende Tumoren, bei welchen keine Metastasen zu entstehen pflegen, z. B. ausser dem Ulcus rodens auch Gliome (Borst, S. 74a).

2) S. 156 . 
im Gegensatz zu der embryonalen Entwickelung, das Epithel nicht in Bindegewebe über. Nachdem wir bereits diese Frage gelöst haben (S. 620), ist uns nicht nur die Ursache der Carcinomentwickelung klar, sondern es ist uns dadurch der Weg gezeigt, auf welchem wir der Carcinomentwickelung entgegenwirken können.

Bereits Ackermann ${ }^{1}$ ) hat den Gedanken ausgesprochen, dass aus dem Sarkom nur deswegen kein Fibrom, d. h. keine bindegewebige Geschwulst entstebt, weil den Zellen, infolge von raschem Untergang, keine Zeit zur Weiterentwickelung zu Bindegewebe gegeben wird. Würden wir aber die Nahrungsaufnahme hier verringern können, so würden sich die Sarkomzellen wieder zu Bindegewebszellen umwandeln können, ebenso wie sie durch grössere Nahrungszufuhr aus den letzteren entstanden waren ${ }^{2}$ ). Das rasche Wachstum und die Malignität des Sarkoms sei nicht - sagt Ackermann - die Folge einer kleinzelligen Struktur, im Gegensatz zur grosszelligen, sondern gerade der rasche Verlauf hat zur Folge, dass die kleinen Sarkomzellen nicht genügend Zeit für die Annahme grösserer Dimensionen haben. Bei den grosszelligen Sarkomen dagegen, eben weil sie langsam wachsen, haben die Zellen Zeit, eine bedeutende Grösse zu erreichen. "In denjenigen Partien, wo die Organisation des Blastems schnell von statten geht, erlangen die Elemente nicht das letzte Stadium ihrer Entwickelung. Wenn dagegen eine Geschwulst, welche während einiger Zeit lebhaft gewachsen war, langsamer zu wachsen anfängt oder stationär bleibt, können die unvollkommenen Elemente, welche darin enthalten waren, ihre Entwickelung vollenden" (Broca) ${ }^{3}$ ).

3. Bei Entzündung des fibrillären Bindegewebes, also bei grösserem lokalen Nahrungszufluss, verschwinden die Fibrillen, die Zellen werden protoplasmareicher, das Bindegewebe wird also „zellig", die Zellen können sich von ihrer Umgebung loslösen und sogar aktive Bewegungen ausführen. Verschwindet aber die Entzündung oder der Wucherungsreiz, so werden die Zellen wieder sesshaft, verbinden sich untereinander durch Fortsätze, werden protoplasmaürmer und es entstehen wieder Fibrillen und schliesslich wird die alte Form des Bindegewebes wieder hergestellt (v. Hansemann).

4. In einem flachen Hautkrebs erfolgt oft Vernarbung (sogenanntes vernarbendes Epitheliom), dann ist auch das Wachstum eines solchen Krebses ein ausserordentlich langsames - ein Beweis, dass wenn das Wachstum ein langsameres Tempo einschlägt, die Zellen dann veruarben können;

1) Volkmanns Sammlung. No. 233-234 (Chirurgie).

2) Billroth meinte sogar, dass jedes Spindelzellensarkom ein junges Neurom oder Myom sei, da im Embryo keine ähnlichen Spindelzellen im Bindegewebe vorkommen. - Cf. auch S. 620 .

3) Traité de tumeurs. p. 187. 
auch beim Carcinoma atrophicans (cicatriciens) mammae, welches ja langsam wächst, bildet sich Bindegewebe.

5. Bekanntlich entsteht aus Lipom eine zelluläre Variation (cfr. S. 613) dadurch, dass die Fettzellen in ihre embryologische Vorstufe, d. h. in die Schleimzellen entdifferenziert werden - es gibt daher keine andere zelluläre Variation des Lipoms, als nur Myxosarkom. Ebenso können sich wieder Schleimzellen in Fettzellen umwandeln (Kölliker) ${ }^{2}$ ).

Auf Grund aller dieser Ueberlegungen müssen wir zu dem Schluss kommen, dass der Uebergang von mehr entdifferenzierten zu phylogenetisch höheren Geweben nicht nur im embryologischen Sinne möglich ist. Schon Virchow änsserte die Ansicht, dass das Corpus luteum ein normales Paradigma eines zellulären Tumors darstelle. Würde hier der Reiz nicht aufhören, so würde sich darans eine maligne Geschwulst entwickeln. Das Wachstum kommt aber im Corpus luteum, trotz der ursprünglich massenhaften Zellvermehrung, endlich zum Stillstand. Der Weg, auf dem hier das Aufbören des Wachstums eingeleitet wird, könnte auch beim Carcinom zum Stillstand der Wuchèrung führen ${ }^{3}$ ). Es würde sich in der Hauptsache darum handeln, das Wachstumstempo zu verlangsamen oder, mit anderen Worten, die Lebensdauer der Carcinomzellen zu verlängern.

Auch die Wachstumsrichtung lässt sich ändern. Schon ảurch die Beeinflussung im eben genannten Sinne wird sie eo ipso sich ändern müssen. Ausserdem wissen wir, dass es viele Faktoren gibt, die eine solche Aenderung bervorrufen können. Fabre-Domergue ${ }^{4}$ ) schlägt vor, den Zytotropismus zu benutzen und mittelst Elektrizität eine Aenderung der Wachstumsrichtung in den Krebszellen hervorzurufen, um auf diese Weise ihrer "Désorientation" entgegenzuwirken. Es ist auch bekannt, dass man bei Pluteuslarven durch Aenderung der osmotischen Verhältnisse (z. B. durch Zusatz von gewissen Salzen) oder durch Erhöhung der Temperatur - die lnvagination des Epithels ganz unterdrücken und sogar das Entoderm nach aussen wachsen lassen kann (Herbst, Driesch, Helene King). Driesch ${ }^{1}$ ) erhielt statt einer Gastrula durch Invagination einen Auswuchs

1) Mitt. aus der Zool. Station zu Neapel. Bd. XI.

2) Mikrosk. Anat. II, I. S. 19. Fig. 92.

3) Loeb gibt an, dass unbefruchtete Seeigeleier sich parthogenetisch bis zu Pluteuslarven weiter entwickeln, wenn sie mit $\mathrm{MgCl}_{2}$ behandelt werden. Herbst (Arch. f. Entw.-Mech. 1896, S. 482, 497) fand, dass bei Asterias durch Rhodankalium die Urdarmbildung unterdrückt wird, so dass sich auf dem Blastulastadium Mesenchym bildet. Das spricht ebenfalls für die chemische Natur des Entwickelungsreizes (Hertwig, Lubarsch, Zur Lehre von Geschwülsten und Infektion. 1899, S. 45).

4) Les cancers épithéliaux. Paris 1898. 
der Blastula in entgegengesetzter Richtung und zwar durch Einwirkung von einer Temperatur von $30^{\circ} \mathrm{C}$. (bei Sphaerechinus).

Ebenso kann man bei Farnen, indem man die Beleuchtungsverhältnisse ändert, die Bildung von Archägonien und Antheridien auf der Oberfläche ihrer Protballien hervorrufen, während normal diese Organe sich hier, wie bekannt, auf der Unterfäche der Prothallien bilden (Loeb) (cf. S. 610). - Bei Aenderung der Sauerstoffquantität kann man bei einigen Schimmelpilzen, z. B. beim Mukor, die Form seines Thallus ändern; der Thallus verliert dann seine fibrilläre Form und dissoziiert sich, indem er der Hefe ähnlich wird (Loeb). Bei Bruchstücken von Polypen gelingt es Köpfe zu entwickeln. Befestigt man solche Stücke in der Art, dass beide Enden vom Wasser umspült werden, so bildet sich nach Loeb am oralen als auch am aboralen Pol ein Köpfchen. Daraus geht hervor, dass man durch mechanische Verletzung der Gewebe an dem aboralen Pol eine höhere Differenzierung erreichen könne, als das normaler Weise geschehen sein würde ${ }^{2}$ ).

Durch verschiedene chemische und andere Mittel lässt sich also die Entwickelung beschleunigen oder verlangsamen. So z. $\mathrm{B}$. $\mathrm{KCl}$, Aether wirken derartig, dass dabei die Eutwickelung als verspätet erscheint. Loeb und Lewis bewiesen, dass durch Erhöhung der Meerwasseralkalinität die Entwickelung beschleunigt werden kann, wobei es sich nicht etwa um Neutralisierung einer im Entwickelungsverlauf produzierten stärkeren Säure handelt.

Die Praxis hat gelehrt, dass alkalische Mittel, in starker Konzentration, lokal angewendet, günstig den Verlauf von Carcinom beeinflussen. Diese Wirkung ist natürlich nur der ätzenden Kraft der konzentrierten Alkalien zuzuschreiben, indem das Carcinom dabei einfach verätzt wird. W. A. Freund ${ }^{3}$ ), von dieser Tatsache ausgehend, hat, wabrscheinlich in der Annahme, dass dabei nicht die Verätzung, sondern das Alkali als solches die Hauptsache sei, eine Durchspülung des Carcinomgewebes mit einem Alkali von geringer Konzentration mit Hilfe des von Jacoby verbesserten Durchspülungsapparates vorgenommen. Die Zusammensetzung dieser schwach alkalischen Lösung war $0,6 \mathrm{NaCl}+0,2$ Zucker $+0,5$ Soda +10 Blutegelkopfextrakt. Die Durcbspülung wurde ausgeführt unter $120-200 \mathrm{~mm} \mathrm{Hg-Druck}$ bei $72-90$ PulsschIägen. Das Resultat war, wie von unserem Gesichtspunkte aus vorauszuseben gewesen wäre, wenig ermunternd. Denn aus den Versuchen Loebs u. A. geht hervor, dass eine Erhöhung der Alkaleszenz das Wachstum befördert und die günstigen Erfolge

1) Untersuch. zur physiol. Morphologie der Tiere. I.

2) Jickeli, S. 87.

3) Beitr. zur Geburtsh. und Gynäkologie. 1898. Bd. I. S. 371. 
nach Einwirkung der Alkalien auf Carcinome waren nur der durch ibre starke Konzentration hervorgerufenen Nekrose zuzuschreiben. Schwach alkalische Mittel würden im Gegenteil das Wachstum nur befördern, wie es in der Tat Freund auch angibt ${ }^{x}$ ). Dagegen würde aus Gründen, die auf S. 609 angegeben worden sind, eben ein leicht saures Medium das Wachstum verlangsamen müssen. Wir verdanken jedenfalls Freund die kühne Methode der Durcbspülung beim Carcinom und es würde sich wohl lohnen, ähnliche Durchispülungsversuche mit schwach sauren Flüssigkeiten anzusstellen. Natürlich wäre einstweilen vor allem an Hautcarcinome zu denken. Würden wir aber diese auf dem bezeichneten Wege zur Heilung bringen, so würde das einen grossen und fundamentalen Fortschritt bedeuten. Beim Magencarcinom würde von diesem Gesichtspunkte aus vielleicht die Anwendung einer verdünnten Säure event. im Verein mit Entfermung des Sauerstoffs (z. B. durch reduzierende Mittel [cf. S. 609]) angezeigt $\operatorname{sein}^{2}$ ).

Moore hat bei allen Carcinomen, nicht nur des Magens, sondern auch andere: Organe eine Verminderung (resp. vollständige Abwesenheit) der $\mathrm{HCl}$ im Magen gefunden. Er schreibt das der Zunahme der Blutalkaleszenz zu. Die Abnahme bedeutet eine Vermehrung der Konzentration von Hydroxylionen und Verminderung der Konzentration von Wasserstoffionen ${ }^{3}$ ).

Die Wirkung dieser Mittel würde ałso in der Hauptsache eine Verlangsamung des Wachstums hervorrufen müssen. Ich habe bereits gezeigt ${ }^{4}$, dass die biologische Therapie des Carcinoms bestehen muss in Verminderung der Nahrungszufuhr (unter gleicbzeitiger Verminderung des Reizes, als den Nahrungszutluss befördernden Mittels), in Verminderung der Temperatur und des Wassergehaltes. Alle diese Mittel wirken makrobiotisch, indem sie die Lebensdauer "der Zellen verlängern. Wir wissen ja, dass durch Wasserentziehung und Temperaturerniedrigung bis zu subnormalen Werten manche niedere Tiere und Pflanzen (Moose, Flechten, Algen u. a.) noch nach 10 Jahren wiederbelebt werden können („vita occulta"), während ihre normale Lebensdauer im Wasser nur wenige Tage oder Wochen beträgt ${ }^{5}$ ). Bei einer Wärme, die einige Grade geringer als $28^{\circ}$ ist, stirbt

1) S. 383.

2) Vergl. meine Arbeit: Das Verbalten der Cholerabazillen in aëroben und anaëroben Kulturen. Arch. f. Hygiene. Bd. XXI. S. 310.

3) On the absence or marked diminution of free $\mathrm{HCl}$ in the gastric content in malignant diseases of organes other then the stomach. Royal Society. Sitzung vom 28. Februar und 26. März 1905.

4) Das Carcinom. S. 30.

5) Auch ein Krystall kann in seiner Entwickelung Halt machen. Von seiner 
der Embryo (bei Hühnern) nicht ab, sondern entwickelt sich nur langsamer; dann folgt ein noch tieferer Grad der Temperatur, welcber obne Weiterbildung das Leben doch erhält ${ }^{1}$ ). Baer fand auch, dass in allen Eiern im Juli (bei ansehnlicher Hitze im Freien) in einer nach Norden liegenden Stube, in welcher überdies zur Abkühlung die Fenster während der Nacht immer offen standen, der Embryo nach Verlauf von 24 Stunden nie abgestorben war, sondern der Herzschlag in sehr langsamen Zwischenräumen, zuweilen von weniger einer Minute, in anderen Fällen von fünf und mehr Minuten fortbestand ${ }^{2}$ ). Barfurth ${ }^{3}$ ) erzielte bei Quappen von Rana fusca durch Halten in kühlem Wasser Formen, bei denen im Oktober nicht einmal die Hinterglieder in Entfaltung gelangt waren, während die Tiere bei gewöhnlicher Temperatur im Freien schon im Mai verwandelt waren. Kölliker und Koller ${ }^{4}$ ) haben die Methode der protrahierten Bebrütung hei niederer Temperatur angewendet, um physiologisch zeitlich nahe bei einander gelegene Stadien auseinander zu ziehen. Dieser Methode verdanken wir die schönsten Aufschlüsse über die Ursprungstätte des Primitivstreifens und der Gastrulationsausstülpung bei Vögeln.

Mlan wird natürlich den Kopf schütteln und sagen: was für einzellige oder niedere Tiere und Pflanzen gilt, gilt nicht für den höheren Organismus. Aber schon die Makrobiotiker haben stets das Moment hervorgehoben: wenig Nahrung. End sie waren es, von denen der Gedanke ausging (Maupertius, Franklin), das Leben des Menschen durch Unterbrechung zu verlängern. Ich brauche hier nur an den Winterschlaf zu erinnern, den sogar Säugetiere aufweisen. Einzelne Käfer können mehrere (bis 6!) Jahre lang ohne Nahrung am Leben bleiben, während sie unter normalen Verbältnissen reichlich Nahrung zu sich nehmen (Weissman, S. 55). Schon Spallanzani im XVIII. Jahrhundert und später Duméril im Jahre 1852 haben gezeigt, dass man einen Frosch langsam einfrieren lassen kann, so dass er durch und durch steinhart wird und keine Spur von Leben mehr zeigt. Taut man ihn vorsichtig auf, so Jebt er wieder. Die Herabsetzung der vitalen Vorgänge ist nur die Folge der Temperaturerniedrigung, da sie zu jeder Jahreszeit hervorgerufen werden kann, wenn nur die Umgebung und das Tier sich genügend abkühlen würden. Man kann den Axolotl durch Aenderung der äusseren Verhältnisse, z. B. durch

Erstarrung erwacht, führt er, den indischen Fakiren ähnlich, seinen Lebenscyklus weiter.

1) Ba er, S. 3.

2) Ibid. S. 4.

3) Archiv f. mikrosliop. Anatomie. Bd. 29.

4) Sitzungsber. d. K. Akad. d. Wiss. zu Wien. Bd. 80. Abt. III. Beitr. zur Kenntnis des Hühnerlieimes im Beginn der Bebrütung. 
die Möglichkeit aufs Trockne zu steigen, in einen Salamander verwandeln und so das Tier nach Belieben auf seiner niedrigeren Entwickelungsstufe halten oder seine Entwickelung weiter führen. Es lassen sich gewisse niedere Tiere (Fliegen, Bienen, Schmetterlinge) durch Verhinderung. der Paarung am Leben bis zum nächsten Frübjahr erhalten, während sie sonst bald nach der Paarung sterben. Ebenso kann man unbefruchtete Seesterneier, die sonst innerhalb 12 Stunden im Seewasser absterben, am Leben erhalten, z. B. durch Verhinderung der Reifung, welche nach Loeb1) durch Hydroxyline zu Stande kommt. Die Neotenie (Boas), d. h. die Rückkehr von komplizierten zu primitiven Zuständen gibt uns ein Beispiel, wie die Geschlechtsreife in eine frühere Periode verlegt wird; aber auch das Gegenteil, wie wir sehen, dürfte nicht unwahrseheinlich sein, und man kann wohl annehmen, dass unter ganz bestimmten Bedingungen die Geschlechtsreife auch in ein späteres Alter verschoben wird. So werden Frauen später geschlechtsreif, wenn sie vom Süden nach Norden kommen ${ }^{2}$ ). Spät geschlechtsreife Tiere besitzen in sich die günstigsten Bedingungen zur Hervorbringung stark progredienter phylogenetischer Reihen (Mehnert).

Die Lebensdauer einer Pflanze stellt keine konstante Grösse vor; es kann eine einjährige Pflanze unter veränderten äusseren Bedingungen sich in eine mehrjährige und umgekehrt eine mehrjährige in eine einjährige umwandeln. Melilotus dentata, Echinospermum lappula sterben im ersten Jahre bald nachdem sie aufgeblüht sind und Früchte gebracht haben, während sie sonst zwei Jahre leben. So stirbt die Aloë (Agave americana) in ihrem Vaterlande nach 5-10 Jahren, d. h. nach der Blüte- und Fruchtreife, wäbrend sie in unseren Gärten erst nach 50-100 Jahren stirbt. Es lässt sich die Lebensdauer künstlich verlängern. Rizinuspflanze wächst in ihrem Vaterlande in Baumgestalt, während sie bei uns, kultiviert in Gärten, zu den einjährigen gehört; die aromatische Reseda kann beim Kultivieren im Garten zu einer straucbartigen Pflanze werden, während sie in natürlichem Zustande, im Freien, eine einjährige Pflanze vorstellt. Die bekannte Belle de nuit (mirabilis jalapa) wächst bei uns als einjährige Pflanze, während sie in ihrem Vaterlande, in Amerika, einige Jahre blüht. Pflanzen, die bei uns einjährig sind, werden im Norden mehrjährig, weil sie im Laufe eines Sommers ihre Entwickelung nicht vollenden können.

Wenn es also möglich ist, die Lebensdauer ganzer Organismen zu verlängern, warum sollte es nicht gelingen, die Lebensdauer einzelner Zellen $z u$ verlängern? Mit Verlängerung der Lebensdauer der Carcinomzellen

1) Archiv für die gesamte Physiologie. Bd. CXIII. 1902. S. 59, 1 u. 2.

2) Mohnert, Biomechanik. S. 161. 
würde aber auch die Bösartigkeit des Carcinoms verschwinden, denn langsames Wachstum schliesst im allgemeinen Bösartigkeit aus.

Mit einem Wort, Temperaturerniedrigung, Verminderung der Wasserzufuhr ${ }^{1}$ ), der Nahrungszufuhr, Anwendung von schwachen Säuren, Aenderung der osmotischen Verhältnisse, der Beleuchtungsbedingungen, der Sauerstoffzufuhr - das sind die Mittel, auf die die Biologie hinweist. Den Tierzüchtern ist schon seit langer Zeit der Einfluss der Nahrung auf die Entwickelung bestimmter Organe bekannt; dies findet in der Praxis vielfache Verwendung. Schwefel oder schwefelhaltige Substanzen spornen bei direkter Applikation oder mässiger interner Einnahme die epithelialen Hautdecken und deren nähere Derivate, insbesondere das Haarkleid, zu besonders reger Entwickelung an. As, Mn, P zeigen bei geringen Dosen ähnliche Wirkungen. P-Salze ind -Dämpfe und Perlmutterstaub führen zu beschleunigtem Knochenwachstum. Es ist jedenfalls der grössten Beachtung wert, dass gerade diejenigen Faktoren, die das Carcinom zur Ausheilung bringen können, wie z. B. das Arsen und die Röntgenisierung, auch die Entstehung von Carcinom herbeiführen können ${ }^{2}$ ).

Schütz ${ }^{3}$ ) glaubt aus seiner Theorie die Berechtigung für die Therapie des Carcinoms ableiten zu dürfen, die in der Absperrung der für die Krebszellen nötigen roten Blutkörperchen bestehen soll (wie es schon von Dawbarn in New-York versucht worden ist). Der Körper erzeugt - sagt Y $o \mathrm{hr}^{4}$ ) - beim Krebs ähnliche Schutzstoffe, wie etwa nach Einführung fremder Blutkörperchen. Die entzündliche Reaktion in der Umgebung des Krebsgewebes, die Phagozytose, die Hyperplasie des benachbarten Lymphgewebes, die reichliche Stromabildung einerseits, die regressiven Metamorphosen (fettige, colloide Entartung der Krebszellen), die Verkalkung andererseits werden im Sinne einer Heilung gedeutet. Auch eine Verminderung der Hyperämie und Reizung kann einen Rückgang des carcinomatösen Prozesses bedingen (z. B. nach einfachen Laparotomien oder Magendarmoperationen). Heftige Entzïndungen in der Geschwulst selbst (Erysipel, Fäulnisprozesse) können deren Zerfall verursachen.

Wir besitzen Mittel, welche in einzelnen Organen, sogar in einzelnen Teilen derselben, lokale Anämie und Hyperämie hervorzurufen imstande sind. Vielleicht würde es ebenso gelingen, die Wasseraufnahme zu verringern und die Temperatur lokal herabzusetzen.

Wir wissen auch, dass nach Gastroenterostomie, nach Laparotomie, nach Kastration (Oophorektomie), Erysipel usw., Carcinome oft von selbst

1) Vielleicht auch durch lokale Anwendung von wasserentziehenden Mitteln.

2) Auch Sarkome verschwinden bekanntlich oft unter Arsenbehandlung.

3) Archiv für Dermatologie und Syphilis. LXII. 1902. S. 91.

4) Therapeutische Monatshefte. XVII. 11, 12. 1903. 
verschwinden und dass überhaupt lebhafte Vorgänge an anderen Körperteilen das Carcinom zum Schwinden bringen können. Man könnte also durch häufig wiederholte multiple Eingriffe an anderen Körperteilen das Wachstum des Carcinoms zum Sistieren zu bringen versuchen. „Die Erzeugung einer tüchtigen Eiterung und Wundgranulation kann beim Carcinom möglicherweise viel nützlicher sein, als eine prima intentio und als treffliche Nachoperation wirken", sagt Waldeyer1).

Es wäre auch inbetracht zu ziehen die Nachahmung des Prozesses, welcher z. B. in der Mamma zur Rückbildung des Carcinoms, und zwar infolge von Kompression der Gefässe durch Bindegewebe führt. Nach Beatson ${ }^{2}$ ) wirkt die Kastration bei Frauen günstig auf den Verlauf des Mammacarcinoms dadurch, dass das Stroma zunimmt und das Epithel atrophiert und fettig degeneriert.

Als weiterer Anhaltspunkt für die biologische Therapie des Carcinoms wäre hervorzuheben, dass bei gewissen Zellarten keine zelluläre Neubildung vorkommt, so z. B. bei Ganglienzellen (cf. S. 624). Es ist nicht unwahrscheinlich, dass dem Unterschiede im anatomischen Bau zwischen diesen Zellen und den Zellen anderer Gewebe auch ein Unterschied in der chemischen Zusammensetzung entspricht. Vielleicht wären daher Einspritzungen von Substanzen aus diesem Gewebe oder derartige Immunisierungssera beim Carcinom von Nutzen. Versuche in dieser Richtung wurden von mir bereits an Kranken angestellt, wie es scheint mit günstigem Resultat. Auch in diagnostischer Hinsicht scheint dieses Prinzip von Bedeutung zu $\operatorname{sein}^{3}$ ). Wenn es einmal so hoch organisierte Wesen geben wird, welche (der im umgekehrten Verhältnis zur phylogenetischen Dignität stehenden Regenerationsfähigkeit gemäss) keine Regenerationseigenschaften mehr besitzen werden, wenn diese Wesen also den höchsten Grad der Integration erlangen werden, dann wird es auch kein Carcinom mehr geben.

Die Schwierigkeit der Therapie liegt vor allem darin, dass die allerersten Anfänge des Carcinoms wenig bestimmte Symptome bieten.

1) Volkmannn's Sammlung. S. 196.

2) Lancet. 1905. I. 11./III. No. X. p. 644.

3) Näheres darüber werde ich an anderer Stelle mitteilen. 